\title{
Sliding Intermittent Control for BAM Neural Networks with Delays
}

\author{
Jianqiang Hu, ${ }^{1}$ Jinling Liang, ${ }^{1}$ Hamid Reza Karimi, ${ }^{2}$ and Jinde Cao ${ }^{1,3}$ \\ ${ }^{1}$ Department of Mathematics, Research Center for Complex Systems and Network Sciences, Southeast University, \\ Nanjing 210096, China \\ ${ }^{2}$ Department of Engineering, Faculty of Engineering and Science, University of Agder, 4898 Grimstad, Norway \\ ${ }^{3}$ Department of Mathematics, King Abdulaziz University, Jeddah 21589, Saudi Arabia
}

Correspondence should be addressed to Jinling Liang; jinlliang@gmail.com

Received 9 May 2013; Accepted 29 May 2013

Academic Editor: Zidong Wang

Copyright (C) 2013 Jianqiang Hu et al. This is an open access article distributed under the Creative Commons Attribution License, which permits unrestricted use, distribution, and reproduction in any medium, provided the original work is properly cited.

This paper addresses the exponential stability problem for a class of delayed bidirectional associative memory (BAM) neural networks with delays. A sliding intermittent controller which takes the advantages of the periodically intermittent control idea and the impulsive control scheme is proposed and employed to the delayed BAM system. With the adjustable parameter taking different particular values, such a sliding intermittent control method can comprise several kinds of control schemes as special cases, such as the continuous feedback control, the impulsive control, the periodically intermittent control, and the semi-impulsive control. By using analysis techniques and the Lyapunov function methods, some sufficient criteria are derived for the closed-loop delayed BAM neural networks to be globally exponentially stable. Finally, two illustrative examples are given to show the effectiveness of the proposed control scheme and the obtained theoretical results.

\section{Introduction}

Since the bidirectional associative memory (BAM) neural networks were first proposed by Kosko [1] which are well known as the extension of the unidirectional autoassociators such as the Hopfield neural networks, they have been widely studied due to their extensive applications such as pattern recognition, signal or image processing, solving optimization problems, and automatic control [2-7]. Later, constant delays are introduced in [8] to the BAM neural networks, and it is proved that the delayed versions of the neural networks are significant for handling certain motion-related optimization problems [9]. For more results concerning the dynamical behaviors of the BAM neural networks with delays, we refer to $[10,11]$.

In practice, most of the neural networks are unstable or convergent with a rate far less than the requirement. Under such cases we need to try to stabilize them or speed up the convergence rate of the neural system in order to make the system work more efficiently. Therefore, the designing of appropriate control input becomes extremely urgent. When it comes to the problem of stabilizing a nonlinear system, it is natural to consider the feedback strategies. There are two basic kinds of feedback control: the state feedback control and the output feedback control. When referring to the control methods, different kinds of schemes have been utilized to stabilize the nonlinear system such as static feedback control [12], delayed feedback control [13], adaptive control [14], fuzzy control [15], sampled control [16], sliding mode control [17], and random control $[18,19]$. In terms of the control time, the controllers are classified with continuous control and discontinuous control. Compared with the continuous control, the discontinuous control including the impulsive control $[20,21]$ and the intermittent control $[22,23]$ has attracted much more attention, and it is very effective, practical, and applicable in many areas, especially in secure communication [24].

In the literature, the impulsive neural networks have been extensively studied from the following two aspects: either the system is subject to the impulsive state displacements at fixed time instants or the system is imposed by external impulsive control [25]. The main idea of periodically intermittent 
control [26] is that when the system signal becomes weak to a low level, the external control will be imposed to supplement the loss of signal; after some period of time the external control is stopped; in the next control period the external control is needed again. Compared with the method of periodically intermittent control, the system with the impulsive control is activated only at some isolated time moments. Both the impulsive control and the intermittent control have their own benefits and disadvantages. The main difference between these two control techniques lies in the length of control period; the former has zero duration, while the later has a nonzero control width. Meanwhile, the cost of the intermittent control is much higher.

The aim of this paper is to design a sliding intermittent controller by combining the advantages of both the impulsive control and the periodically intermittent control. More specifically, in one control period, we will impose the continuous state feedback control at the preceding control width and the impulsive control in the latter control width. The sliding intermittent control method is very flexible and could achieve the expected control performance. The sketch of such a controller is shown in Figure 1. Motivated by the name of the slide rheostat in the physical electronic circuitry, we named such a joint controller as the sliding intermittent controller.

In this paper, we will investigate the exponential stability problem of the delayed BAM neural networks under the proposed sliding intermittent control. The closed-loop neural system becomes a switched network where the switching rules are dependent on the time index. To the best of the authors' knowledge, this is the first time in the literature to consider such a joint controller. The rest of the paper is organized as follows. In Section 2, the model discussed in this paper and the novel sliding intermittent control idea are introduced, and some preliminaries are also given. In Section 3, several sufficient criteria are established to ensure the delayed BAM neural networks to be exponentially stable under the sliding intermittent control scheme. Meanwhile, several particular cases are discussed. In Section 4, two illustrative examples are given to demonstrate the effectiveness of the proposed results. And finally, the paper is concluded in Section 5 .

\section{Problem Formulation and Some Preliminaries}

The delayed bidirectional associative memory (BAM) neural networks have been investigated in $[8,9]$ as follows:

$$
\begin{gathered}
\dot{w}_{i}(t)=-a_{i} w_{i}(t)+\sum_{j=1}^{m} p_{i j} \tilde{f}_{j}\left(z_{j}\left(t-\tau_{i j}\right)\right)+c_{i}, \quad i \in \mathscr{I}_{n}, \\
\dot{z}_{j}(t)=-b_{j} z_{j}(t)+\sum_{i=1}^{n} q_{j i} \tilde{\mathcal{G}}_{i}\left(w_{i}\left(t-\sigma_{j i}\right)\right)+d_{j}, \quad j \in \mathscr{I}_{m},
\end{gathered}
$$

where the index set $\mathscr{I}_{n}=\{1,2, \ldots, n\}, \mathscr{I}_{m}=\{1,2, \ldots$, $m\} ; w_{i}(t), z_{j}(t) \in \mathbb{R}$ are the activations of the $i$ th neuron

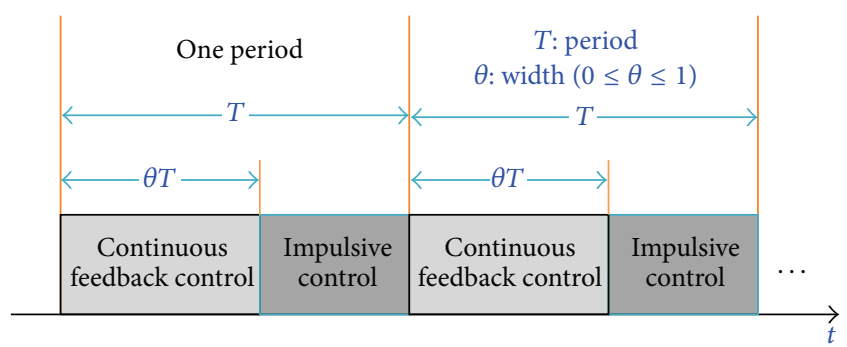

FIGURE 1: Sketch map of the sliding intermittent control.

and the $j$ th neuron, respectively; $a_{i}, b_{j}$ are positive constants denoting the rates with which the cells $i$ and $j$ reset their potential to the resting states when isolated from the other cells and inputs; time delays $\tau_{i j}$ and $\sigma_{j i}$ are nonnegative constants corresponding to the finite speeds of axonal signal transmission with $\tau^{*}=\max _{i, j}\left\{\tau_{i j}, \sigma_{j i}\right\} ; p_{i j}$ and $q_{j i}$ are the delayed connection weights denoting the strengths of connectivity between the cells $j$ and $i ; c_{i}$ and $d_{j}$ denote, respectively, the $i$ th and the $j$ th components of an external input source introduced from the network outside to the cells $i$ and $j ; \tilde{f}_{j}$ and $\tilde{g}_{i}$ are bounded nonlinear activation functions, and throughout this paper they are assumed to satisfy the following conditions:

$$
\begin{aligned}
& \left|\tilde{f}_{j}\left(\nu_{1}\right)-\tilde{f}_{j}\left(v_{2}\right)\right| \leq L_{j}^{f}\left|v_{1}-v_{2}\right|, \\
& \left|\tilde{g}_{i}\left(v_{1}\right)-\tilde{g}_{i}\left(v_{2}\right)\right| \leq L_{i}^{g}\left|v_{1}-v_{2}\right|,
\end{aligned}
$$

where $\nu_{1}, v_{2} \in \mathbb{R}$ and $i \in \mathscr{I}_{n}, j \in \mathscr{I}_{m}$; positive scalars $L_{j}^{f}$, $L_{i}^{g}$ are known.

Remark 1. The above conditions are general in the literature to study the existence and uniqueness of the equilibrium for the delayed BAM neural networks (1) without assuming the activation functions to be monotonic or differentiable [2729].

Letting $\left(w^{*}, z^{*}\right)$ with $w^{*}=\left(w_{1}^{*}, w_{2}^{*}, \ldots, w_{n}^{*}\right)^{T} \in$ $\mathbb{R}^{n}$ and $z^{*}=\left(z_{1}^{*}, z_{2}^{*}, \ldots, z_{m}^{*}\right)^{T} \in \mathbb{R}^{m}$ be an equilibrium of the system (1) and denoting $x_{i}(t)=w_{i}(t)-w_{i}^{*}, y_{j}(t)=z_{j}(t)-$ $z_{j}^{*}$, the equilibrium of the network (1) can be transformed to the origin of the following system:

$$
\begin{array}{ll}
\dot{x}_{i}(t)=-a_{i} x_{i}(t)+\sum_{j=1}^{m} p_{i j} f_{j}\left(y_{j}\left(t-\tau_{i j}\right)\right), & i \in \mathscr{I}_{n}, \\
\dot{y}_{j}(t)=-b_{j} y_{j}(t)+\sum_{i=1}^{n} q_{j i} g_{i}\left(x_{i}\left(t-\sigma_{j i}\right)\right), & j \in \mathscr{I}_{m},
\end{array}
$$

where $f_{j}\left(y_{j}\right)=\tilde{f}_{j}\left(y_{j}+z_{j}^{*}\right)-\tilde{f}_{j}\left(z_{j}^{*}\right)$ and $g_{i}\left(x_{i}\right)=\tilde{g}_{i}\left(x_{i}+w_{i}^{*}\right)-$ $\tilde{g}_{i}\left(w_{i}^{*}\right)$.

It is generally known that, under some specific cases such as the abrupt changes of system parameters or the occurrence of time delays, the network may present unstable dynamics such as bifurcation, oscillation, divergence, or instability. In 
this paper, we will focus on the unstable delayed BAM neural system (3). To stabilize the network (3), the novel sliding intermittent control scheme is imposed as follows:

$$
\begin{aligned}
& \dot{x}_{i}(t)=-a_{i} x_{i}(t)+\sum_{j=1}^{m} p_{i j} f_{j}\left(y_{j}\left(t-\tau_{i j}\right)\right)+u_{i}^{(1)}(t), \\
& t \in[l T,(l+\theta) T), \\
& \dot{x}_{i}(t)=-a_{i} x_{i}(t)+\sum_{j=1}^{m} p_{i j} f_{j}\left(y_{j}\left(t-\tau_{i j}\right)\right), \\
& \quad t \in[(l+\theta) T,(l+1) T), t \neq t_{k}, \\
& \Delta x_{i}(t)=I_{k}^{(1)}\left(x_{i}(t)\right), \quad t=t_{k}, \\
& \dot{y}_{j}(t)=-b_{j} y_{j}(t)+\sum_{i=1}^{n} q_{j i} g_{i}\left(x_{i}\left(t-\sigma_{j i}\right)\right)+u_{j}^{(2)}(t), \\
& \dot{y}_{j}(t)=-b_{j} y_{j}(t)+\sum_{i=1}^{n} q_{j i} g_{i}\left(x_{i}\left(t-\sigma_{j i}\right)\right), \\
& t \in[(l+\theta) T,(l+1) T), t \neq t_{k}, \\
& \Delta y_{j}(t)=I_{k}^{(2)}\left(y_{j}(t)\right), \quad t=t_{k},
\end{aligned}
$$

where $i \in \mathscr{I}_{n}, j \in \mathscr{I}_{m} ; l \in \mathbb{N}_{0}^{+} \triangleq\{0,1,2, \ldots$,$\} and k \in \mathbb{N}^{+} \triangleq$ $\{1,2, \ldots\}$; constant $\theta \in[0,1]$ and $T$ are scalars denoting the control width and the control period, respectively; $\Delta x_{i}\left(t_{k}\right)=$ $x_{i}\left(t_{k}^{+}\right)-x_{i}\left(t_{k}^{-}\right), \Delta y_{j}\left(t_{k}\right)=y_{j}\left(t_{k}^{+}\right)-y_{j}\left(t_{k}^{-}\right)$and $x_{i}\left(t_{k}^{+}\right)=$ $\lim _{h \rightarrow 0^{+}} x_{i}\left(t_{k}+h\right), x_{i}\left(t_{k}\right)=x_{i}\left(t_{k}^{-}\right)=\lim _{h \rightarrow 0^{-}} x_{i}\left(t_{k}+\right.$ $h), y_{j}\left(t_{k}^{+}\right)=\lim _{h \rightarrow 0^{+}} y_{j}\left(t_{k}+h\right)$, and $y_{j}\left(t_{k}\right)=y_{j}\left(t_{k}^{-}\right)=$ $\lim _{h \rightarrow 0^{-}} y_{j}\left(t_{k}+h\right)$. The impulsive sequence $\left\{t_{k}\right\}_{k=1}^{\infty}$ satisfies

$$
\begin{array}{r}
0<t_{1}<t_{2}<\cdots<t_{k}<\cdots, \lim _{k \rightarrow \infty} t_{k}=\infty, \\
t_{k} \in \bigcup_{l \in \mathbb{N}_{0}^{+}}((l+\theta) T,(l+1) T] .
\end{array}
$$

$u_{i}^{(1)}(t), u_{j}^{(2)}(t)$ are controllers imposed on the system (3) when $t \in[l T,(l+\theta) T)$, and $I_{k}^{(1)}(\cdot), I_{k}^{(2)}(\cdot)$ are the impulsive operators imposed at the impulsive moments $\left\{t_{k}\right\}_{k=1}^{\infty}$. Here we use the linear state feedback and linear impulsive control strategies; that is,

$$
\begin{array}{cc}
u_{i}^{(1)}(t)=k_{i}^{(1)} x_{i}(t), & u_{j}^{(2)}(t)=k_{j}^{(2)} y_{j}(t), \\
I_{k}^{(1)}\left(x_{i}\left(t_{k}\right)\right)=h_{i k}^{(1)} x_{i}\left(t_{k}\right), & I_{k}^{(2)}\left(y_{j}\left(t_{k}\right)\right)=h_{j k}^{(2)} y_{j}\left(t_{k}\right),
\end{array}
$$

where $k_{i}^{(1)}, k_{j}^{(2)}, h_{i k}^{(1)}$, and $h_{j k}^{(2)}$ are gain constants.

Remark 2. The sliding intermittent control idea is illustrated in Figure 1 with the adjustable parameter $\theta$ (i.e., the control width $\theta$ shown in Figure 1). The closed-loop system (4) can be the continuous controlled neural networks $(\theta=1)$, the impulsive controlled neural networks $(\theta=0)$, or the hybrid controlled neural networks $(0<\theta<1)$.

The system (4) is supplemented with initial function given by $x_{i}(s)=\varphi_{i}(s), y_{j}(s)=\psi_{j}(s),-\tau^{*} \leq s \leq 0$, where $\varphi(s)=\left(\varphi_{1}(s), \varphi_{2}(s), \ldots, \varphi_{n}(s)\right)^{T} \in C\left(\left[-\tau^{*}, 0\right], \mathbb{R}^{n}\right), \psi(s)=$ $\left(\psi_{1}(s), \psi_{2}(s), \ldots, \psi_{m}(s)\right)^{T} \in C\left(\left[-\tau^{*}, 0\right], \mathbb{R}^{m}\right)$, and $C\left(\left[-\tau^{*}, 0\right]\right.$, $\left.\mathbb{R}^{n}\right)$ represents the set of all $n$-dimensional continuous functions defined on the interval $\left[-\tau^{*}, 0\right]$. Obviously, the solution of (4) is piecewise left-hand continuous with possible discontinuity at $t=t_{k}$ for $k \in \mathbb{N}^{+}$.

The following definition and lemmas are introduced before we give the main results of the paper.

Definition 3. The system (4) is said to be globally exponentially stable if there exist constants $\alpha>0, \beta>0$ such that for all $t>0$,

$$
\begin{aligned}
& \sum_{i=1}^{n}\left|x_{i}(t)\right|+\sum_{j=1}^{m}\left|y_{j}(t)\right| \\
& \quad \leq \beta e^{-\alpha t} \sup _{-\tau^{*} \leq s \leq 0}\left(\sum_{i=1}^{n}\left|\varphi_{i}(s)\right|+\sum_{j=1}^{m}\left|\psi_{j}(s)\right|\right)
\end{aligned}
$$

holds for all $\varphi(\cdot) \in C\left(\left[-\tau^{*}, 0\right], \mathbb{R}^{n}\right)$ and $\psi(\cdot) \in C\left(\left[-\tau^{*}, 0\right]\right.$, $\left.\mathbb{R}^{m}\right)$.

Lemma 4 (see [30]). Let $V(\cdot):\left[t_{0}-\tau, \infty\right) \rightarrow[0, \infty)$ be a continuous function such that

$$
\dot{V}(t) \leq-a V(t)+b \bar{V}(t)
$$

is satisfied for $t \geq t_{0}$. If $a>b>0$, then

$$
V(t) \leq \bar{V}\left(t_{0}\right) e^{-\lambda\left(t-t_{0}\right)}, \quad t \geq t_{0},
$$

where $\bar{V}(t)=\sup _{t-\tau \leq s \leq t} V(s), \lambda>0$ is the unique positive real root of the equation $-a+\lambda+b e^{\lambda \tau}=0$.

Lemma 5 (see [31]). Let $q \geq 0, \tau>0, \mu_{k}>0(k=1,2, \ldots)$, and $p$ be constants, and assume that $V(t)$ is a piecewise continuous nonnegative function satisfying

$$
\begin{gathered}
D^{+} V(t) \leq p V(t)+q \bar{V}(t), \quad t \geq t_{0}, t \neq t_{k} \\
V\left(t_{k}^{+}\right) \leq \mu_{k} V\left(t_{k}\right), \quad k=1,2, \ldots
\end{gathered}
$$

If there exists constant $\beta$ such that

$$
\frac{\ln \mu_{k}}{t_{k}-t_{k-1}} \leq \beta, \quad p+d q+\beta<0,
$$

hold for $k=1,2, \ldots$, then

$$
V(t) \leq d \bar{V}\left(t_{0}\right) e^{-\lambda\left(t-t_{0}\right)},
$$

where $\bar{V}(t)=\sup _{t-\tau \leq s \leq t} V(s), d=\sup _{k}\left\{e^{\beta\left(t_{k}-t_{k-1}\right)}\right.$, $\left.e^{-\beta\left(t_{k}-t_{k-1}\right)}\right\}<\infty$, and $\lambda$ is the unique positive root of the equation $\lambda+p+d q e^{\lambda \tau}+\beta=0$. 


\section{Main Results}

In this section, some sufficient criteria will be given based on the sliding intermittent control scheme. First, the global exponential stability of the closed-loop hybrid neural networks (4) is analyzed, and then several criteria are obtained by setting different parameters in the sliding intermittent controller.

Theorem 6. Assume the upper bound delay $\tau^{*}<\min \{\theta T$, $(1-\theta) T\}$ and the external imposed impulsive strengths satisfy $h_{i k}^{(1)}, h_{j k}^{(2)} \neq-1$. Under the sliding intermittent control, the closed-loop control system (4) is globally exponentially stable if there exist constants $\beta$ and $k_{i}^{(1)}, k_{j}^{(2)}$ such that the following conditions hold:

(i)

$$
\begin{aligned}
& a_{i}-k_{i}^{(1)}>L_{i}^{g} \sum_{j=1}^{m}\left|q_{j i}\right|, \quad i \in \mathscr{I}_{n}, \\
& b_{j}-k_{j}^{(2)}>L_{j}^{f} \sum_{i=1}^{n}\left|p_{i j}\right|, \quad j \in \mathscr{I}_{m},
\end{aligned}
$$

(ii)

(iii)

$$
\begin{array}{r}
\frac{\ln \mu_{k+1}}{t_{k+1}-t_{k}} \leq \beta, \quad \rho+d \tilde{b}_{1}+\beta<0, \\
k \in \mathbb{N}^{+} \backslash\left\{i_{l} \mid l=0,1,2, \ldots\right\},
\end{array}
$$

$$
\tilde{a}_{1}>\widetilde{b}_{1}, \quad \varrho \triangleq \eta+\lambda_{1}(\theta-\gamma)-\lambda_{2}(\theta+\gamma)-\frac{\ln d}{T}>0,
$$

where $\tilde{a}_{1}=\min _{i, j}\left\{a_{i}-k_{i}^{(1)}-\eta, b_{j}-k_{j}^{(2)}-\eta\right\} ; \widetilde{b}_{1}=\max _{i}\left\{L_{i}^{g}\right.$ $\left.\sum_{j=1}^{m}\left|q_{j i}\right| e^{\eta \sigma_{j i}}\right\}+\max _{j}\left\{L_{j}^{f} \sum_{i=1}^{n}\left|p_{i j}\right| e^{\eta \tau_{i j}}\right\} ; \mu_{k}=\max _{i, j}\{\mid 1+$ $\left.h_{i k}^{(1)}|| 1+,h_{j k}^{(2)} \mid\right\} ; \rho=\max _{i, j}\left\{\eta-a_{i}, \eta-b_{j}\right\} ; d=\sup _{k}\left\{e^{\beta\left(t_{k+1}-t_{k}\right)}\right.$, $\left.e^{-\beta\left(t_{k+1}-t_{k}\right)}\right\} ; t_{i_{l}}$ is used to denote the last impulsive moment on the interval $[(l+\theta) T,(l+1) T)$ with $t_{i_{l}}<(l+1) T-\tau^{*} ; \gamma=$ $\tau^{*} / T ; 0<\eta \leq \eta^{*} ; \eta^{*}=\min _{i, j}\left\{\theta_{i}^{*}, \vartheta_{j}^{*} \mid F_{i}\left(\theta_{i}^{*}\right)=0, G_{j}\left(\vartheta_{j}^{*}\right)=\right.$ 0\} with functions $F_{i}(\cdot), G_{j}(\cdot)$ defined as

$$
\begin{gathered}
F_{i}\left(\theta_{i}\right)=a_{i}-k_{i}^{(1)}-\theta_{i}-\sum_{j=1}^{m}\left|q_{j i}\right| L_{i}^{g} e^{\theta_{i} \sigma_{j i}}, \quad \theta_{i} \in[0, \infty), \\
G_{j}\left(\vartheta_{j}\right)=b_{j}-k_{j}^{(2)}-\vartheta_{j}-\sum_{i=1}^{n}\left|p_{i j}\right| L_{j}^{f} e^{\vartheta_{j} \tau_{i j}}, \quad \vartheta_{j} \in[0, \infty),
\end{gathered}
$$

and $\lambda_{1}, \lambda_{2}$ are, respectively, the unique positive real root of the equations $-\tilde{a}_{1}+\lambda_{1}+\widetilde{b}_{1} e^{\lambda_{1} \tau^{*}}=0$ and $\lambda_{2}+\rho+d \tilde{b}_{1} e^{\lambda_{2} \tau^{*}}+\beta=0$.
More specifically, we have the following inequality:

$$
\begin{aligned}
& \sum_{i=1}^{n}\left|x_{i}(t)\right|+\sum_{j=1}^{m}\left|y_{j}(t)\right| \\
& \quad \leq M e^{-\varrho t} \sup _{-\tau^{*} \leq s \leq 0}\left(\sum_{i=1}^{n}\left|\varphi_{i}(s)\right|+\sum_{j=1}^{m}\left|\psi_{j}(s)\right|\right), \quad t>0,
\end{aligned}
$$

with $M=\max \left\{e^{\lambda_{1}(\theta-\gamma) \theta T}, e^{\lambda_{2}(1-\theta-\gamma) \theta T+(1-\theta) \ln d}\right\}$.

Proof. For the functions $F_{i}(\cdot)$ and $G_{j}(\cdot)$ defined in (16), from the condition (13), it is clear that

$$
\begin{aligned}
& F_{i}(0)=a_{i}-k_{i}^{(1)}-\sum_{j=1}^{m}\left|q_{j i}\right| L_{i}^{g}>0, \\
& G_{j}(0)=b_{j}-k_{j}^{(2)}-\sum_{i=1}^{n}\left|p_{i j}\right| L_{j}^{f}>0 .
\end{aligned}
$$

Since $F_{i}(\cdot)$ and $G_{j}(\cdot)$ are continuous on $[0, \infty)$ and $\lim _{\theta_{i} \rightarrow \infty} F_{i}\left(\theta_{i}\right)=-\infty, \lim _{\vartheta_{j} \rightarrow \infty} G_{j}\left(\vartheta_{j}\right)=-\infty$, there must exist constants $\theta_{i}^{*}>0$ and $\vartheta_{j}^{*}>0$ such that $F_{i}\left(\theta_{i}^{*}\right)=$ 0 and $G_{j}\left(\vartheta_{j}^{*}\right)=0$. By setting $\eta^{*}=\min \left\{\theta_{1}^{*}, \theta_{2}^{*}, \ldots\right.$, $\left.\theta_{n}^{*}, \vartheta_{1}^{*}, \vartheta_{2}^{*}, \ldots, \vartheta_{m}^{*}\right\}$, one obtains that, for any $0<\eta \leq \eta^{*}$,

$$
\begin{aligned}
& F_{i}(\eta)=a_{i}-k_{i}^{(1)}-\eta-\sum_{j=1}^{m}\left|q_{j i}\right| L_{i}^{g} e^{\eta \sigma_{j i}} \geq 0, \\
& G_{j}(\eta)=b_{j}-k_{j}^{(2)}-\eta-\sum_{i=1}^{n}\left|p_{i j}\right| L_{j}^{f} e^{\eta \tau_{i j}} \geq 0 .
\end{aligned}
$$

Now consider the Lyapunov function defined as follows:

$$
V(t)=\sum_{i=1}^{n} u_{i}(t)+\sum_{j=1}^{m} v_{j}(t), \quad t \geq 0
$$

where $u_{i}(t)=e^{\eta t}\left|x_{i}(t)\right|$ and $v_{j}(t)=e^{\eta t}\left|y_{j}(t)\right|$. Obviously, $V(t)$ is a positive definite function for $t \geq 0$.

(1) When $t \in[l T,(l+\theta) T), l \in \mathbb{N}_{0}^{+}$, one is easy to have

$$
\begin{aligned}
D^{+} u_{i}(t) \leq & -\left(a_{i}-k_{i}^{(1)}-\eta\right) u_{i}(t) \\
& +\sum_{j=1}^{m}\left|p_{i j}\right| L_{j}^{f} e^{\eta \tau_{i j}} v_{j}\left(t-\tau_{i j}\right), \\
D^{+} v_{j}(t) \leq & -\left(b_{j}-k_{j}^{(2)}-\eta\right) v_{j}(t) \\
& +\sum_{i=1}^{n}\left|q_{j i}\right| L_{i}^{g} e^{\eta \sigma_{j i}} u_{i}\left(t-\sigma_{j i}\right) .
\end{aligned}
$$


Calculating the upper right Dini derivative of $V(t)$ along the solutions of network (4), from the above inequality, we get

$$
\begin{aligned}
D^{+} V(t) \leq & -\sum_{i=1}^{n}\left(a_{i}-k_{i}^{(1)}-\eta\right) u_{i}(t) \\
& -\sum_{j=1}^{m}\left(b_{j}-k_{j}^{(2)}-\eta\right) v_{j}(t) \\
& +\sum_{i=1}^{n} \sum_{j=1}^{m}\left|p_{i j}\right| L_{j}^{f} e^{\eta \tau_{i j}} v_{j}\left(t-\tau_{i j}\right) \\
& +\sum_{j=1}^{m} \sum_{i=1}^{n}\left|q_{j i}\right| L_{i}^{g} e^{\eta \sigma_{j i}} u_{i}\left(t-\sigma_{j i}\right) \\
\leq & -\widetilde{a}_{1} V(t)+\widetilde{b}_{1}\left(\sup _{t-\tau^{*} \leq s \leq t} V(s)\right)
\end{aligned}
$$

where $\tilde{a}_{1}=\min _{i, j}\left\{a_{i}-k_{i}^{(1)}-\eta, b_{j}-k_{j}^{(2)}-\eta\right\}$ and $\tilde{b}_{1}=\max _{i}\left\{L_{i}^{g}\right.$ $\left.\sum_{j=1}^{m}\left|q_{j i}\right| e^{\eta \sigma_{j i}}\right\}+\max _{j}\left\{L_{j}^{f} \sum_{i=1}^{n}\left|p_{i j}\right| e^{\eta \tau_{i j}}\right\}$. By Lemma 4 , one has

$$
V(t) \leq e^{-\lambda_{1}(t-l T)}\left(\sup _{l T-\tau^{*} \leq s \leq l T} V(s)\right)=e^{-\lambda_{1}(t-l T)} \bar{V}(l T),
$$

$$
t \in[l T,(l+\theta) T)
$$

where $\lambda_{1}$ is the unique positive real root of the equation $-\widetilde{a}_{1}+\lambda_{1}+\widetilde{b}_{1} e^{\lambda_{1} \tau^{*}}=0$.

(2) When $t \in[(l+\theta) T,(l+1) T)$ and $t \neq t_{k}, l \in \mathbb{N}_{0}^{+}, k \in \mathbb{N}^{+}$, it is easy to have

$$
\begin{aligned}
& D^{+} u_{i}(t) \leq-\left(a_{i}-\eta\right) u_{i}(t)+\sum_{j=1}^{m}\left|p_{i j}\right| L_{j}^{f} e^{\eta \tau_{i j}} v_{j}\left(t-\tau_{i j}\right), \\
& D^{+} v_{j}(t) \leq-\left(b_{j}-\eta\right) v_{j}(t)+\sum_{i=1}^{n}\left|q_{j i}\right| L_{i}^{g} e^{\eta \sigma_{j i}} u_{i}\left(t-\sigma_{j i}\right) .
\end{aligned}
$$

Without loss of generality, suppose $t \in\left(t_{k-1}, t_{k}\right], k \leq i_{l}$, where $t_{i_{l}}$ is assumed to be the last impulsive moment on the interval $[(l+\theta) T,(l+1) T)$. It follows from the inequality (24) that

$$
\begin{aligned}
D^{+} V(t) \leq & \sum_{i=1}^{n}\left(\eta-a_{i}\right) u_{i}(t)+\sum_{j=1}^{m}\left(\eta-b_{j}\right) v_{j}(t) \\
& +\sum_{i=1}^{n}\left(L_{i}^{g} \sum_{j=1}^{m} e^{\eta \sigma_{j i}}\left|q_{j i}\right|\right) u_{i}\left(t-\sigma_{j i}\right) \\
& +\sum_{j=1}^{m}\left(L_{j}^{f} \sum_{i=1}^{n} e^{\eta \tau_{i j}}\left|p_{i j}\right|\right) v_{j}\left(t-\tau_{i j}\right) \\
\leq & \rho V(t)+\widetilde{b}_{1}\left(\sup _{t-\tau^{*} \leq s \leq t} V(s)\right)
\end{aligned}
$$

where $\rho=\max _{i, j}\left\{\eta-a_{i}, \eta-b_{j}\right\} ; \widetilde{b}_{1}=\max _{i}\left\{L_{i}^{g} \sum_{j=1}^{m} e^{\eta \sigma_{j i}}\left|q_{j i}\right|\right\}+$ $\max _{j}\left\{L_{j}^{f} \sum_{i=1}^{n} e^{\eta \tau_{i j}}\left|p_{i j}\right|\right\}$.

When $t \in[(l+\theta) T,(l+1) T)$ and $t=t_{k}$,

$$
\begin{aligned}
& x_{i}\left(t_{k}^{+}\right)=x_{i}\left(t_{k}\right)+I_{k}^{(1)}\left(x_{i}\left(t_{k}\right)\right)=\left(1+h_{i k}^{(1)}\right) x_{i}\left(t_{k}\right), \\
& y_{j}\left(t_{k}^{+}\right)=y_{j}\left(t_{k}\right)+I_{k}^{(2)}\left(y_{j}\left(t_{k}\right)\right)=\left(1+h_{j k}^{(2)}\right) y_{j}\left(t_{k}\right) .
\end{aligned}
$$

Considering the condition that $h_{i k}^{(1)}, h_{j k}^{(2)} \neq-1$, we have $\mu_{k}>$ 0 and

$$
\begin{aligned}
u_{i}\left(t_{k}^{+}\right) & =e^{\eta t_{k}^{+}}\left|x_{i}\left(t_{k}^{+}\right)\right| \\
& \leq\left|1+h_{i k}^{(1)}\right| u_{i}\left(t_{k}\right) \leq \mu_{k} u_{i}\left(t_{k}\right), \quad i \in \mathscr{I}_{n}, \\
v_{j}\left(t_{k}^{+}\right) & =e^{\eta t_{k}^{+}}\left|y_{j}\left(t_{k}^{+}\right)\right| \\
& \leq\left|1+h_{j k}^{(2)}\right| v_{j}\left(t_{k}\right) \leq \mu_{k} v_{j}\left(t_{k}\right), \quad j \in \mathscr{I}_{m},
\end{aligned}
$$

which infers that

$$
V\left(t_{k}^{+}\right)=\sum_{i=1}^{n} u_{i}\left(t_{k}^{+}\right)+\sum_{j=1}^{m} v_{j}\left(t_{k}^{+}\right) \leq \mu_{k} V\left(t_{k}\right) .
$$

From condition (14) and Lemma 5, one has

$$
\begin{array}{r}
V(t) \leq d e^{-\lambda_{2}(t-(l+\theta) T)} \bar{V}((l+\theta) T), \\
t \in[(l+\theta) T,(l+1) T),
\end{array}
$$

where $d=\sup _{k}\left\{e^{\beta\left(t_{k}-t_{k-1}\right)}, e^{-\beta\left(t_{k}-t_{k-1}\right)}\right\}$ and $\lambda_{2}$ is the unique positive real root of the equation $\lambda_{2}+\rho+d \widetilde{b}_{1} e^{\lambda_{2} \tau^{*}}+\beta=0$.

(3) Now, we are ready to estimate $V(t)$ based on the inequalities (23) and (29) with the method of induction.

When $t \in[0, \theta T)$, one obtains

$$
V(t) \leq e^{-\lambda_{1} t} \bar{V}(0) .
$$

When $t \in[\theta T, T)$, one can derive

$$
\begin{aligned}
V(t) & \leq d e^{-\lambda_{2}(t-\theta T)} \bar{V}(\theta T) \\
& \leq d e^{-\left[\lambda_{2}(t-\theta T)+\lambda_{1}\left(\theta T-\tau^{*}\right)\right]} \bar{V}(0) .
\end{aligned}
$$

When $t \in[T,(1+\theta) T)$, we have

$$
\begin{aligned}
V(t) & \leq e^{-\lambda_{1}(t-T)} \bar{V}(T) \\
& \leq d e^{-\left[\lambda_{2}\left(T-\tau^{*}-\theta T\right)+\lambda_{1}\left(t-(1-\theta) T-\tau^{*}\right)\right]} \bar{V}(0) .
\end{aligned}
$$

When $t \in[(1+\theta) T, 2 T)$, one can derive

$$
\begin{aligned}
V(t) & \leq d e^{-\lambda_{2}(t-(1+\theta) T)} \bar{V}((1+\theta) T) \\
& \leq d^{2} e^{-\left[\lambda_{2}\left(t-2 \theta T-\tau^{*}\right)+2 \lambda_{1}\left(\theta T-\tau^{*}\right)\right]} \bar{V}(0) .
\end{aligned}
$$


By induction, one can derive the following estimation of $V(t)$ for any integer $l \in \mathbb{N}_{0}^{+}$:

when $t \in[l T,(l+\theta) T)$,

$$
V(t) \leq d^{l} e^{-\left[l \lambda_{2}\left(T-\theta T-\tau^{*}\right)+\lambda_{1}\left(t-l\left(T-\theta T+\tau^{*}\right)\right)\right]} \bar{V}(0),
$$

and when $t \in[(l+\theta) T,(l+1) T)$,

$$
V(t) \leq d^{l+1} e^{-\left[\lambda_{2}\left(t-(l+1) \theta T-l \tau^{*}\right)+(l+1) \lambda_{1}\left(\theta T-\tau^{*}\right)\right]} \bar{V}(0) .
$$

By setting $\gamma=\tau^{*} / T$ and substituting it to the above two inequalities, one has, for $t \in[l T,(l+\theta) T)$,

$$
\begin{aligned}
V(t) & \leq d^{l} e^{-\left[\lambda_{2} l T(1-\theta-\gamma)+\lambda_{1}(t-l T)+\lambda_{1}(\theta-\gamma) l T\right]} \bar{V}(0) \\
& \leq d^{l} e^{\left[\lambda_{2}(\theta+\gamma) l T-\lambda_{1}(\theta-\gamma) l T\right]} \bar{V}(0) \\
& \leq d^{l} e^{\left[\lambda_{2}(\theta+\gamma) t+\lambda_{1}(\theta-\gamma)(-t+\theta T)\right]} \bar{V}(0) \\
& =d^{l} e^{-\left[\lambda_{1}(\theta-\gamma)-\lambda_{2}(\theta+\gamma)\right] t} e^{\lambda_{1}(\theta-\gamma) \theta T} \bar{V}(0) \\
& \leq e^{(\ln d / T) t} e^{-\left[\lambda_{1}(\theta-\gamma)-\lambda_{2}(\theta+\gamma)\right] t} e^{\lambda_{1}(\theta-\gamma) \theta T} \bar{V}(0) \\
& \leq e^{-\left[\lambda_{1}(\theta-\gamma)-\lambda_{2}(\theta+\gamma)-((\ln d) / T)\right] t} M \bar{V}(0),
\end{aligned}
$$

and, for $t \in[(l+\theta) T,(l+1) T)$,

$$
\begin{aligned}
V(t) & \leq d^{l+1} e^{-\left[\lambda_{2}(t-(l+1) \theta T-l \gamma T)+\lambda_{1}(\theta-\gamma)(l+1) T\right]} \bar{V}(0) \\
& \leq d^{l+1} e^{\left[\lambda_{2} \theta T+\lambda_{2} l T(\theta+\gamma)-\lambda_{1}(\theta-\gamma) t\right]} \bar{V}(0) \\
& \leq d^{l+1} e^{\left[\lambda_{2} \theta T+\lambda_{2}(t-\theta T)(\theta+\gamma)-\lambda_{1}(\theta-\gamma) t\right]} \bar{V}(0) \\
& =d^{l+1} e^{\left[\lambda_{2}(\theta+\gamma) t-\lambda_{1}(\theta-\gamma) t\right]+\lambda_{2} \theta T(1-\theta-\gamma)} \bar{V}(0) \\
& \leq e^{((t / T)-\theta+1) \ln d} e^{-\left[\lambda_{1}(\theta-\gamma)-\lambda_{2}(\theta+\gamma)\right] t} e^{\lambda_{2} \theta T(1-\theta-\gamma) \bar{V}(0)} \\
& \leq e^{-\left[\lambda_{1}(\theta-\gamma)-\lambda_{2}(\theta+\gamma)-((\ln d) / T)\right] t} M \bar{V}(0)
\end{aligned}
$$

Therefore, we have

$$
V(t) \leq e^{-\left[\lambda_{1}(\theta-\gamma)-\lambda_{2}(\theta+\gamma)-((\ln d) / T)\right] t} M \bar{V}(0), \quad t>0,
$$

which means that, for any $t>0$,

$$
\begin{aligned}
& \sum_{i=1}^{n}\left|x_{i}(t)\right|+\sum_{j=1}^{m}\left|y_{j}(t)\right| \\
& \quad \leq e^{-\left[\eta+\lambda_{1}(\theta-\gamma)-\lambda_{2}(\theta+\gamma)-((\ln d) / T)\right] t} M \bar{V}(0),
\end{aligned}
$$

where $M=\max \left\{e^{\lambda_{1}(\theta-\gamma) \theta T}, e^{\lambda_{2}(1-\theta-\gamma) \theta T+(1-\theta) \ln d}\right\}$. And the conclusion that the origin of network (4) is exponentially stable. The proof is complete.

Remark 7. In the above Theorem 6, the control width $\theta \in$ $(0,1)$ which does not include the boundary cases. If the parameter $\theta \rightarrow 0$, the controlled system approximates to impulsive neural networks. If the parameter $\theta \rightarrow 1$, the controlled system approximates to continuous neural networks. And the controlled system can be handled, respectively, by the methods of the impulsive system and the continuous system. In order to avoid such boundary cases, usually we can take $\theta=0.5$; that is, the periodically intermittent controller and the impulsive controller play a significant role in the process of control; this results a switched neural networks.

Remark 8. Most of the literatures [28, 32] concerning the global exponential stability of the delayed BAM neural networks with impulses have focused on the stable system. Namely, without the impulsive disturbance, the original neural networks are stable, and under the impulsive disturbance the system can still be kept stable with particular conditions. While, in this article, the impulses can be viewed as either control input or impulsive disturbance, at the same time, the original system is not required to be stable initially.

If adjustable parameter $\theta=1$, the controlled system turns out to be the following model; that is, the original unstable system (3) is controlled with the continuous feedback controller. Under such case, the conditions in the following proposition will guarantee the closed-loop system to be globally exponentially stable:

$$
\begin{aligned}
\dot{x}_{i}(t)= & -a_{i} x_{i}(t)+\sum_{j=1}^{m} p_{i j} f_{j}\left(y_{j}\left(t-\tau_{i j}\right)\right) \\
& +u_{i}^{(1)}(t), \quad i \in \mathscr{I}_{n}, \\
\dot{y}_{j}(t)= & -b_{j} y_{j}(t)+\sum_{i=1}^{n} q_{j i} g_{i}\left(x_{i}\left(t-\sigma_{j i}\right)\right) \\
& +u_{j}^{(2)}(t), \quad j \in \mathscr{I}_{m} .
\end{aligned}
$$

Proposition 9. Under the continuous feedback control scheme, the origin of the closed-loop control system (40) is globally exponentially stable if there exist constants $k_{i}^{(1)}$ and $k_{j}^{(2)}$ such that

$$
\begin{aligned}
& a_{i}-k_{i}^{(1)}>L_{i}^{g} \sum_{j=1}^{m}\left|q_{j i}\right|, \quad i \in \mathscr{I}_{n}, \\
& b_{j}-k_{j}^{(2)}>L_{j}^{f} \sum_{i=1}^{n}\left|p_{i j}\right|, \quad j \in \mathscr{I}_{m} .
\end{aligned}
$$

Furthermore, one has the following inequality:

$$
\begin{aligned}
& \sum_{i=1}^{n}\left|x_{i}(t)\right|+\sum_{j=1}^{m}\left|y_{j}(t)\right| \\
& \leq\left(\widetilde{b}_{1} \tau^{*}+1\right) e^{-\eta t} \sup _{-\tau^{*} \leq s \leq 0}\left(\sum_{i=1}^{n}\left|\varphi_{i}(s)\right|\right. \\
&\left.+\sum_{j=1}^{m}\left|\psi_{j}(s)\right|\right), \quad t>0,
\end{aligned}
$$


where the parameters $\eta$ and $\widetilde{b}_{i}$ are consistent with the ones in Theorem 6.

Proof. Consider the Lyapunov function defined as follows:

$$
\begin{aligned}
& W(t)=V(t) \\
& \qquad \sum_{i=1}^{n} \sum_{j=1}^{m}\left(\left|p_{i j}\right| L_{j}^{f} e^{\eta \tau_{i j}} \int_{t-\tau_{i j}}^{t} v_{j}(s) \mathrm{d} s\right. \\
& \left.\quad+\left|q_{j i}\right| L_{i}^{g} e^{\eta \sigma_{j i}} \int_{t-\sigma_{j i}}^{t} u_{i}(s) \mathrm{d} s\right), \quad t \geq 0 .
\end{aligned}
$$

The upper right Dini derivative of $W(t)$ with respect to time $t$ along the solutions of the network (40) can be calculated as follows:

$$
\begin{aligned}
D^{+} W(t)=\sum_{i=1}^{n} D^{+} u_{i}(t)+\sum_{j=1}^{m} D^{+} v_{j}(t) & \\
& +\sum_{i=1}^{n} \sum_{j=1}^{m}\left|p_{i j}\right| L_{j}^{f} e^{\eta \tau_{i j}}\left[v_{j}(t)-v_{j}\left(t-\tau_{i j}\right)\right] \\
& +\sum_{j=1}^{m} \sum_{i=1}^{n}\left|q_{j i}\right| L_{i}^{g} e^{\eta \sigma_{j i}}\left[u_{i}(t)-u_{i}\left(t-\sigma_{j i}\right)\right] \\
\leq & -\sum_{i=1}^{n}\left(a_{i}-k_{i}^{(1)}-\eta-\sum_{j=1}^{m}\left|q_{j i}\right| L_{i}^{g} e^{\eta \sigma_{j i}}\right) u_{i}(t) \\
& -\sum_{j=1}^{m}\left(b_{j}-k_{j}^{(2)}-\eta-\sum_{i=1}^{n}\left|p_{i j}\right| L_{j}^{f} e^{\eta \tau_{i j}}\right) v_{j}(t) \\
= & -\sum_{i=1}^{n} F_{i}(\eta) u_{i}(t)-\sum_{j=1}^{m} G_{j}(\eta) v_{j}(t) \leq 0
\end{aligned}
$$

which means that $W(t) \leq W(0)$. Hence we have $\sum_{i=1}^{n}\left|x_{i}(t)\right|+$ $\sum_{j=1}^{m}\left|y_{j}(t)\right| \leq\left(\widetilde{b}_{1} \tau^{*}+1\right) e^{-\eta t} \bar{V}(0)$, and this completes the proof.

If the impulsive strengths $h_{i k}^{(1)}=h_{j k}^{(2)} \equiv 0$, namely, there are no impulsive controls on the latter control interval in each control period, which means the closed-loop system is only subject to the continuous feedback control in the preceding control width of each control period. Such a case is then reduced to the pure periodically intermittent control, and the neural network system (4) turns into the following controlled neural network (45). The conditions in the following proposition will guarantee the closed-loop system to be globally exponentially stable. In order to obtain the main result, the following lemma is given firstly

$$
\begin{array}{r}
\dot{x}_{i}(t)=-a_{i} x_{i}(t)+\sum_{j=1}^{m} p_{i j} f_{j}\left(y_{j}\left(t-\tau_{i j}\right)\right)+u_{i}^{(1)}(t), \\
l T \leq t<(l+\theta) T, \\
\dot{x}_{i}(t)=-a_{i} x_{i}(t)+\sum_{j=1}^{m} p_{i j} f_{j}\left(y_{j}\left(t-\tau_{i j}\right)\right), \\
(l+\theta) T \leq t<(l+1) T, \\
\dot{y}_{j}(t)=-b_{j} y_{j}(t)+\sum_{i=1}^{n} q_{j i} g_{i}\left(x_{i}\left(t-\sigma_{j i}\right)\right)+u_{j}^{(2)}(t), \\
l T \leq t<(l+\theta) T,
\end{array}
$$

$$
\begin{array}{r}
\dot{y}_{j}(t)=-b_{j} y_{j}(t)+\sum_{i=1}^{n} q_{j i} g_{i}\left(x_{i}\left(t-\sigma_{j i}\right)\right), \\
(l+\theta) T \leq t<(l+1) T .
\end{array}
$$

Lemma 10 (see [23]). Let $V(\cdot):\left[t_{0}-\tau, \infty\right) \rightarrow[0, \infty)$ be a continuous function such that

$$
\dot{V}(t) \leq a V(t)+b\left(\sup _{t-\tau \leq s \leq t} V(s)\right)
$$

is satisfied for $t \geq t_{0}$. If $a>0, b>0$, then

$$
\begin{aligned}
V(t) & \leq \sup _{t-\tau \leq s \leq t} V(s) \\
& \leq\left(\sup _{t_{0}-\tau \leq s \leq t_{0}} V(s)\right) e^{(a+b)\left(t-t_{0}\right)}, \quad t \geq t_{0} .
\end{aligned}
$$

Proposition 11. Assuming the upper bound delay $\tau^{*}<$ $\min \{\theta T,(1-\theta) T\}$, under the periodically intermittent control, the closed-loop control system (45) is globally exponentially stable if the control gains $k_{i}^{(1)}$ and $k_{j}^{(2)}$ satisfy the following conditions:

(i)

$$
\begin{aligned}
& a_{i}-k_{i}^{(1)}>L_{i}^{g} \sum_{j=1}^{m}\left|q_{j i}\right|, \quad i \in \mathscr{I}_{n}, \\
& b_{j}-k_{j}^{(2)}>L_{j}^{f} \sum_{i=1}^{n}\left|p_{i j}\right|, \quad j \in \mathcal{I}_{m},
\end{aligned}
$$

(ii)

$\widetilde{a}_{1}>\widetilde{b}_{1}, \quad \eta+\lambda_{1}(\theta-\gamma)-\left(\widetilde{\rho}+\widetilde{b}_{1}\right)(1-\theta)>0$. 
Moreover, we have

$$
\begin{aligned}
\sum_{i=1}^{n}\left|x_{i}(t)\right|+\sum_{j=1}^{m}\left|y_{j}(t)\right| \\
\leq \bar{M} e^{-\left[\eta+\lambda_{1}(\theta-\gamma)-\left(\tilde{\rho}+\widetilde{b}_{1}\right)(1-\theta)\right] t} \\
\quad \times \sup _{-\tau^{*} \leq s \leq 0}\left(\sum_{i=1}^{n}\left|\varphi_{i}(s)\right|+\sum_{j=1}^{m}\left|\psi_{j}(s)\right|\right), \quad t>0,
\end{aligned}
$$

where $\tilde{\rho}=\max _{i, j}\left\{\eta-a_{i}, \eta-b_{j}, \kappa\right\}$, and $\kappa$ is any positive constant; $\widetilde{M}=e^{\lambda_{1} \theta T(\theta-\gamma)}$, and the parameters $\tilde{a}_{1}, \widetilde{b}_{1}, \eta$, $\lambda_{1}$, and $\gamma$ are consistent with those in Theorem 6.

Proof. Considering the same Lyapunov function as that in Theorem 6, by similar analytical technique, one can get the following results on the control period and the control width as follows.

(1) When $t \in[l T,(l+\theta) T), l \in \mathbb{N}_{0}^{+}$, we have $D^{+} V(t) \leq$ $-\widetilde{a}_{1} V(t)+\widetilde{b}_{1} \bar{V}(t)$. By Lemma 4 , one obtains that, for any $t \in[l T,(l+\theta) T), V(t) \leq e^{-\lambda_{1}(t-l T)} \bar{V}(l T)$.

(2) When $t \in[(l+\theta) T,(l+1) T), l \in \mathbb{N}_{0}^{+}$, we have $D^{+} V(t) \leq \widetilde{\rho} V(t)+\widetilde{b}_{1} \bar{V}(t)$. By Lemma 10, it is derived that, for any $t \in[(l+\theta) T,(l+1) T), V(t) \leq$ $e^{\left(\widetilde{\rho}+\widetilde{b}_{1}\right)(t-(l+\theta) T)} \bar{V}((l+\theta) T)$.

From the above inequality relationships, by similar estimation procedure, we can get the following conclusion:

$$
\begin{gathered}
V(t) \leq e^{-\lambda_{1}(t-l T)+l\left(\widetilde{\rho}+\widetilde{b}_{1}\right)(1-\theta) T-\lambda_{1} l\left(\theta-\tau^{*}\right)} \bar{V}(0), \\
t \in[l T,(l+\theta) T), \\
V(t) \leq e^{-\lambda_{1}(l+1)\left(\theta T-\tau^{*}\right)+l\left(\tilde{\rho}+\widetilde{b}_{1}\right)(1-\theta) T+\left(\widetilde{\rho}+\widetilde{b}_{1}\right)(t-l T-\theta T)} \bar{V}(0), \\
t \in[(l+\theta) T,(l+1) T) .
\end{gathered}
$$

By the notational expression $\tau^{*}=\gamma T$, one can further obtain

$$
\begin{gathered}
V(t) \leq e^{\left[-\lambda_{1}(\theta-\gamma)+\left(\widetilde{\rho}+\widetilde{b}_{1}\right)(1-\theta)\right] t} e^{\lambda_{1} \theta T(\theta-\gamma)} \bar{V}(0), \\
t \in[l T,(l+\theta) T), \\
V(t) \leq e^{\left[-\lambda_{1}(\theta-\gamma)+\left(\widetilde{\rho}+\widetilde{b}_{1}\right)(1-\theta)\right] t} \bar{V}(0), \\
t \in[(l+\theta) T,(l+1) T) .
\end{gathered}
$$

Hence it follows that, for any $t>0$,

$$
\begin{aligned}
\sum_{i=1}^{n}\left|x_{i}(t)\right|+\sum_{j=1}^{m}\left|y_{j}(t)\right| \\
\leq \bar{M} e^{-\left[\eta+\lambda_{1}(\theta-\gamma)-\left(\widetilde{\rho}+\widetilde{b}_{1}\right)(1-\theta)\right] t} \\
\quad \times \sup _{-\tau^{*} \leq s \leq 0}\left(\sum_{i=1}^{n}\left|\varphi_{i}(s)\right|+\sum_{j=1}^{m}\left|\psi_{j}(s)\right|\right),
\end{aligned}
$$

and this completes the proof.
If the adjustable parameter $\theta=0$, that is, the original unstable system (3) is subjected to the impulsive controller, then system (4) becomes the following impulsive neural networks (54). Under such a case, it would be natural to assume that the frequency of the impulses should not be too low; that is, some restrictions on the impulsive periods and the impulsive strengths are needed. The conditions in the following proposition will guarantee the closed-loop system (54) to be globally exponentially stable:

$$
\begin{array}{r}
\dot{x}_{i}(t)=-a_{i} x_{i}(t)+\sum_{j=1}^{m} p_{i j} f_{j}\left(y_{j}\left(t-\tau_{i j}\right)\right), \\
t \geq 0, t \neq t_{k}, k \in \mathbb{N}^{+}, \\
\Delta x_{i}\left(t_{k}\right)=I_{k}^{(1)}\left(x_{i}\left(t_{k}\right)\right), \quad k \in \mathbb{N}^{+}, i \in \mathscr{I}_{n}, \\
\dot{y}_{j}(t)=-b_{j} y_{j}(t)+\sum_{i=1}^{n} q_{j i} g_{i}\left(x_{i}\left(t-\sigma_{j i}\right)\right), \\
t \geq 0, t \neq t_{k}, k \in \mathbb{N}^{+}, \\
\Delta y_{j}\left(t_{k}\right)=I_{k}^{(2)}\left(y_{j}\left(t_{k}\right)\right), \quad k \in \mathbb{N}^{+}, j \in \mathscr{I}_{m} .
\end{array}
$$

Proposition 12. Assume the external imposed impulsive strengths $h_{i k}^{(1)}, h_{j k}^{(2)} \neq-1$. The origin of the closed-loop control system (54) is globally exponentially stable if there exists constant $\beta$ such that, for $k=1,2, \ldots$, the following conditions hold:

$$
\frac{\ln \mu_{k}}{t_{k}-t_{k-1}} \leq \beta, \quad \rho+d \widetilde{b}_{1}+\beta<0 .
$$

More specifically, we have

$$
\begin{aligned}
\sum_{i=1}^{n}\left|x_{i}(t)\right|+\sum_{j=1}^{m}\left|y_{j}(t)\right| & \\
\leq d e^{-\left(\eta+\lambda_{2}\right) t} \sup _{-\tau^{*} \leq s \leq 0} & \left(\sum_{i=1}^{n}\left|\varphi_{i}(s)\right|\right. \\
& \left.+\sum_{j=1}^{m}\left|\psi_{j}(s)\right|\right), \quad t>0,
\end{aligned}
$$

where the parameters $\mu_{k}, \rho, d, \widetilde{b}_{1}$, and $\eta$ are consistent with those in Theorem 6.

Proof. Considering the same Lyapunov function as that in Theorem 6 , by similar analytical technique, one can get the following results on the impulsive interval and the impulse moments as follows.

(1) When $t \in\left(t_{k-1}, t_{k}\right]$, the upper right Dini derivative of $V(t)$ along the solution of (54) is depicted as $D^{+} V(t) \leq \rho V(t)+\widetilde{b}_{1}\left(\sup _{t-\tau^{*} \leq s \leq t} V(s)\right)$.

(2) When $t=t_{k}, k \in \mathbb{N}^{+}, V\left(t_{k}^{+}\right) \leq \mu_{k} V\left(t_{k}\right)$. 
From Lemma 5 and the conditions in (55), it follows that

$$
V(t) \leq d\left(\sup _{0-\tau^{*} \leq s \leq 0} V(s)\right) e^{-\lambda_{2}(t-0)},
$$

which means

$$
\begin{aligned}
& \sum_{i=1}^{n}\left|x_{i}(t)\right|+\sum_{j=1}^{m}\left|y_{j}(t)\right| \\
& \quad \leq e^{-\eta t} d e^{-\lambda_{2} t} \bar{V}(0) \leq d e^{-\left(\eta+\lambda_{2}\right) t} \bar{V}(0), \quad t>0
\end{aligned}
$$

and the proof is completed.

If the continuous feedback control gains $k_{i}^{(1)}=k_{j}^{(2)} \equiv$ 0 , that is, there is no feedback on the preceding control interval in a control period, and only impulsive control is imposed on the latter control interval, this means the system is only under the piecewise impulsive (not the uniformly distributed) control. As for such case, the impulsive system is reduced to the following one:

$$
\begin{aligned}
& \dot{x}_{i}(t)=-a_{i} x_{i}(t)+\sum_{j=1}^{m} p_{i j} f_{j}\left(y_{j}\left(t-\tau_{i j}\right)\right), \\
& t \in[l T,(l+\theta) T) \cup\left\{[(l+\theta) T,(l+1) T) \backslash\left\{t \neq t_{k}\right\}_{k=1}^{\infty}\right\}, \\
& \Delta x_{i}(t)=I_{k}^{(1)}\left(x_{i}(t)\right), \quad t=t_{k}, \\
& \dot{y}_{j}(t)=-b_{j} y_{j}(t)+\sum_{i=1}^{n} q_{j i} g_{i}\left(x_{i}\left(t-\sigma_{j i}\right)\right), \\
& t \in[l T,(l+\theta) T) \cup\left\{[(l+\theta) T,(l+1) T) \backslash\left\{t \neq t_{k}\right\}_{k=1}^{\infty}\right\}, \\
& \Delta y_{j}(t)=I_{k}^{(2)}\left(y_{j}(t)\right), \quad t=t_{k} .
\end{aligned}
$$

From (59), it is noticed that the occurrence of the impulses is not uniformly distributed since the impulses never occur on the interval $[l T,(l+\theta) T), l \in \mathbb{N}_{0}^{+}$, whereas they frequently occur on the interval $((l+\theta) T,(l+1) T), l \in \mathbb{N}_{0}^{+}$. By observing the proof of Proposition 12, the result is somewhat more conservative especially when the control period $T$ is very large and the control width $\theta$ approaches to one. In order to describe the conservatism for such case, we will utilize the notation of average impulsive interval proposed in [21] to characterize the occurrence frequency of the impulses. The definition of average impulsive interval and the corresponding impulsive differential inequality are given firstly.

Definition 13 ([21] average impulsive interval). The average impulsive interval of the impulsive sequence $\left\{t_{k}\right\}_{k=1}^{\infty}$ is equal to $T_{a}$ if there exist positive integer $N_{0}$ and positive number $T_{a}$ such that

$$
N\left(\kappa_{2}, \kappa_{1}\right) \geq \frac{\kappa_{2}-\kappa_{1}}{T_{a}}-N_{0}, \quad \forall \kappa_{2} \geq \kappa_{1} \geq 0,
$$

where $N\left(\kappa_{2}, \kappa_{1}\right)$ denotes the number of impulsive times of the impulsive sequence $\left\{t_{k}\right\}_{k=1}^{\infty}$ on the interval $\left(\kappa_{1}, \kappa_{2}\right)$.
Lemma 14 (see Lakshmikantham et al., Theorem 1.4.1, [33, $34])$. Let $P C\left(\mathbb{R}_{+}, \mathbb{R}\right)\left(P C^{1}\left(\mathbb{R}_{+}, \mathbb{R}\right)\right)$ denote the set of piecewise continuous (piecewise continuously differentiable) functions with first kind of discontinuities at $t_{k}, k=1,2, \ldots$, from $\mathbb{R}_{+}$to $\mathbb{R}$. If the following conditions hold,

$$
\begin{array}{r}
m(t) \in P C^{1}\left(\mathbb{R}_{+}, \mathbb{R}\right) \text { is left continuous at } t_{k}, \\
k=1,2, \ldots, \\
D^{+} m(t) \leq p(t) m(t)+q(t), \quad t \geq t_{0}, \quad t \neq t_{k}, \\
m\left(t_{k}^{+}\right) \leq d_{k} m\left(t_{k}\right)+b_{k}, \quad k=1,2, \ldots,
\end{array}
$$

where $p(t), q(t) \in P C\left(\mathbb{R}_{+}, \mathbb{R}\right), d_{k} \geq 0$ and $b_{k}$ are real constants, then

$$
\begin{aligned}
m(t) \leq & m\left(t_{0}\right) \prod_{t_{0}<t_{k}<t} d_{k} \exp \left(\int_{t_{0}}^{t} p(s) d s\right) \\
& +\sum_{t_{0}<t_{k}<t}\left(\prod_{t_{0}<t_{j}<t} d_{j} \exp \left(\int_{t_{k}}^{t} p(s) d s\right)\right) b_{k} \\
& +\int_{t_{0}}^{t} \prod_{s<t_{k}<t} d_{k} \exp \left(\int_{s}^{t} p(\xi) d \xi\right) q(s) d s, \quad t \geq t_{0} .
\end{aligned}
$$

Lemma 15. Let $q \geq 0, \tau>0,0<\mu<1$, and $p$ be constants, and assume that $V(t)$ is a piecewise continuous nonnegative function satisfying

$$
\begin{gathered}
D^{+} V(t) \leq p V(t)+q \bar{V}(t), \quad t \geq t_{0}, \quad t \neq t_{k}, \\
V\left(t_{k}^{+}\right) \leq \mu V\left(t_{k}\right), \quad k=1,2, \ldots,
\end{gathered}
$$

and the average impulsive interval of the impulsive sequence $\left\{t_{k}\right\}_{k=1}^{\infty}$ is equal to $T_{a}$. If the following inequality holds,

$$
p+\frac{\ln \mu}{T_{a}}+\mu^{-N_{0}} q<0
$$

then one has

$$
V(t) \leq \mu^{-N_{0}} \bar{V}\left(t_{0}\right) e^{-\lambda\left(t-t_{0}\right)},
$$

where $\bar{V}(t)=\sup _{t-\tau \leq s \leq t} V(s), \lambda$ is the unique positive root of the equation $\lambda+p+\left(\ln \mu / T_{a}\right)+\mu^{-N_{0}} q e^{\lambda \tau}=0$. 
Proof. By Lemma 14 and the definition of average impulsive interval, it follows from (63) that, for $t \geq t_{0}$,

$$
\begin{gathered}
V(t) \leq \bar{V}\left(t_{0}\right) \mu^{N\left(t, t_{0}\right)} e^{p\left(t-t_{0}\right)}+\int_{t_{0}}^{t} e^{p(t-s)} \mu^{N(t, s)} q \bar{V}(s) \mathrm{d} s \\
\leq \bar{V}\left(t_{0}\right) \mu^{\left(\left(\left(t-t_{0}\right) / T_{a}\right)-N_{0}\right)} e^{p\left(t-t_{0}\right)} \\
\quad+\int_{t_{0}}^{t} e^{p(t-s)} \mu^{\left(\left((t-s) / T_{a}\right)-N_{0}\right)} q \bar{V}(s) \mathrm{d} s \\
\leq \mu^{-N_{0}}\left(\bar{V}\left(t_{0}\right) e^{\left[p+\left(\ln \mu / T_{a}\right)\right]\left(t-t_{0}\right)}\right. \\
\left.+\int_{t_{0}}^{t} e^{\left[p+\left(\ln \mu / T_{a}\right)\right](t-s)} q \bar{V}(s) \mathrm{d} s\right) .
\end{gathered}
$$

Denote $\phi(\lambda)=\lambda+p+\left(\ln \mu / T_{a}\right)+\mu^{-N_{0}} q e^{\lambda \tau}$. Since $\phi(0)=p+$ $\left(\ln \mu / T_{a}\right)+\mu^{-N_{0}} q<0$, and $\lim _{\lambda \rightarrow \infty} \phi(\lambda)=+\infty$ and $\phi^{\prime}(\lambda)=$ $1+\mu^{-N_{0}} q \tau e^{\lambda \tau}>0$, one knows that $\phi(\lambda)=0$ has a unique positive root.

Next, it is claimed that, for all $t>t_{0}$,

$$
V(t)<\mu^{-N_{0}}\left(\sup _{t_{0}-\tau \leq s \leq t_{0}} V(s)\right) e^{-\lambda\left(t-t_{0}\right)} .
$$

When $t \in\left[t_{0}-\tau, t_{0}\right]$,

$$
V(t)<\bar{V}\left(t_{0}\right)<\mu^{-N_{0}} \bar{V}\left(t_{0}\right) e^{-\lambda\left(t-t_{0}\right)} .
$$

Supposing (67) is not always true for $t>t_{0}$, there must exist one time point $t^{*}>t_{0}$ such that

$$
\begin{gathered}
V\left(t^{*}\right)=\mu^{-N_{0}} \bar{V}\left(t_{0}\right) e^{-\lambda\left(t^{*}-t_{0}\right)}, \\
V(t)<\mu^{-N_{0}} \bar{V}\left(t_{0}\right) e^{-\lambda\left(t-t_{0}\right)}, \quad t_{0}-\tau \leq t<t^{*} .
\end{gathered}
$$

From inequalities (66) and (69), one can obtain that

$$
V\left(t^{*}\right)
$$

$$
\begin{aligned}
& \leq \mu^{-N_{0}}\left(\bar{V}\left(t_{0}\right) e^{\left[p+\left(\ln \mu / T_{a}\right)\right]\left(t^{*}-t_{0}\right)}\right. \\
& \left.+\int_{t_{0}}^{t^{*}} e^{\left[p+\left(\ln \mu / T_{a}\right)\right]\left(t^{*}-s\right)} q \bar{V}(s) \mathrm{d} s\right) \\
& <\mu^{-N_{0}}\left(\bar{V}\left(t_{0}\right) e^{\left[p+\left(\ln \mu / T_{a}\right)\right]\left(t^{*}-t_{0}\right)}\right. \\
& \left.+\int_{t_{0}}^{t^{*}} e^{\left[p+\left(\ln \mu / T_{a}\right)\right]\left(t^{*}-s\right)} q \mu^{-N_{0}} \bar{V}\left(t_{0}\right) e^{-\lambda\left(s-\tau-t_{0}\right)} \mathrm{d} s\right) \\
& =\mu^{-N_{0}} \bar{V}\left(t_{0}\right) e^{\left[p+\left(\ln \mu / T_{a}\right)\right]\left(t^{*}-t_{0}\right)} \\
& +q \mu^{-2 N_{0}} \bar{V}\left(t_{0}\right) e^{\lambda \tau} \int_{t_{0}}^{t^{*}} e^{\left[p+\left(\ln \mu / T_{a}\right)\right]\left(t^{*}-s\right)} e^{-\lambda\left(s-t_{0}\right)} \mathrm{d} s .
\end{aligned}
$$

Considering the equality $\psi(\lambda)=\lambda+p+\left(\ln \mu / T_{a}\right)+$ $\mu^{-N_{0}} q e^{\lambda \tau}=0$, one has

$$
\begin{aligned}
V\left(t^{*}\right)< & \mu^{-N_{0}} \bar{V}\left(t_{0}\right) e^{-\lambda\left(t^{*}-t_{0}\right)} \\
& \times\left(e^{\left[-\mu^{-N_{0}} q e^{\lambda \tau}\right]\left(t^{*}-t_{0}\right)}\right. \\
& \left.+q \mu^{-N_{0}} e^{\lambda \tau} \int_{t_{0}}^{t^{*}} e^{\left[-\mu^{-N_{0}} q e^{\lambda \tau}\right]\left(t^{*}-s\right)} \mathrm{d} s\right) \\
= & \mu^{-N_{0}} \bar{V}\left(t_{0}\right) e^{-\lambda\left(t^{*}-t_{0}\right)} e^{-\left[\mu^{-N_{0}} q e^{\lambda \tau}\right] t^{*}} \\
& \times\left(e^{\left[\mu^{-N_{0}} q e^{\lambda \tau}\right] t_{0}}\right. \\
& \left.+e^{\left[\mu^{-N_{0}} q e^{\lambda \tau}\right] t^{*}}-e^{\left[\mu^{-N_{0}} q e^{\lambda \tau}\right] t_{0}}\right) \\
= & \mu^{-N_{0}} \bar{V}\left(t_{0}\right) e^{-\lambda\left(t^{*}-t_{0}\right)}
\end{aligned}
$$

which contradicts with the first inequality of (69). Therefore, the inequality (67) holds, and this completes the proof.

The following proposition will guarantee the system (59) to be globally exponentially stable.

Proposition 16. Assume the external imposed impulsive strengths $h_{i k}^{(1)}, h_{j k}^{(2)} \in(-2,-1) \cup(-1,0)$, and the average impulsive interval of the impulsive sequence $\left\{t_{k}\right\}_{k=1}^{\infty}$ is equal to $T_{a}$. The impulsive system (59) is globally exponentially stable if the following condition holds:

$$
\rho+\frac{\ln \mu}{T_{a}}+\mu^{-N_{0}} \widetilde{b}_{1}<0 .
$$

Moreover, we have

$$
\begin{aligned}
& \sum_{i=1}^{n}\left|x_{i}(t)\right|+\sum_{j=1}^{m}\left|y_{j}(t)\right| \\
& \quad \leq \mu^{-N_{0}} e^{-(\eta+\lambda) t} \sup _{-\tau^{*} \leq s \leq 0}\left(\sum_{i=1}^{n}\left|\varphi_{i}(s)\right|+\sum_{j=1}^{m}\left|\psi_{j}(s)\right|\right), \quad t>0,
\end{aligned}
$$

where $\mu=\sup _{k}\left\{\mu_{k}\right\} \in(0,1)$, and $\mu_{k}, \rho$, and $\tilde{b}_{1}$ are defined as in Theorem 6 and $\lambda$ is the unique positive root of the equation $\lambda+p+\left(\ln \mu / T_{a}\right)+\mu^{-N_{0}} q e^{\lambda \tau}=0$.

Proof. Considering the same Lyapunov function as that in Theorem 6, the following results on the impulsive interval and the impulse moments can be obtained.

(1) When $t \in[l T,(l+\theta) T) \backslash\left\{t_{k}\right\}_{k=1}^{\infty}, l \in \mathbb{N}_{0}^{+}$, the upper right Dini derivative of $V(t)$ along the solutions of (59) is depicted as $D^{+} V(t) \leq \rho V(t)+\widetilde{b}_{1} \bar{V}(t)$.

(2) When $t=t_{k}, k \in \mathbb{N}^{+}, V\left(t_{k}^{+}\right) \leq \mu V\left(t_{k}\right)$. 
From the above two inequality relationships, and Lemma 15, one has

$$
\begin{aligned}
& \sum_{i=1}^{n}\left|x_{i}(t)\right|+\sum_{j=1}^{m}\left|y_{j}(t)\right| \\
& \quad \leq \mu^{-N_{0}} e^{-(\eta+\lambda) t}\left(\sup _{t_{0}-\tau \leq s \leq t_{0}} V(s)\right), \quad t>0,
\end{aligned}
$$

where $\lambda$ is the unique positive root of the equation $\lambda+p+$ $\left(\ln \mu / T_{a}\right)+\mu^{-N_{0}} q e^{\lambda \tau}=0$. The proof is completed.

Remark 17. It should be pointed out that, in the preceding control width of the control period, other kinds of continuous controllers can also be used to achieve the same performance. For example, in [35], the adaptive control scheme has been employed in the control width instead of the continuous state feedback, where the adjusting gains can be designed based on different norms. We can borrow such an idea to the sliding intermittent control design. Moreover, the sliding width parameter can be $\left\{\theta_{i}\right\}$ rather than the fixed width $\theta$, and the period can be $\left\{T_{i}\right\}$ rather than constant $T$. By doing so, we might obtain more general conditions. On the other hand, the phenomena of stochastic nonlinearities are extremely ubiquitous in practical controlled systems [3639]; hence it is more reasonable to consider neural networks with random nonlinearities, and this will be our future works.

\section{Illustrative Example}

In this section, we present some examples to illustrate the applicability and efficiency of the proposed control scheme.

Example 1. Considering the following extensively studied BAM neural system,

$$
\begin{gathered}
\dot{x}(t)=-a x(t)+p f(y(t-\tau)), \\
\dot{y}(t)=-b y(t)+q g(x(t-\sigma))
\end{gathered}
$$

with $a=1.922, p=9.8501, b=1.1631, q=8.2311, \tau=\sigma=$ 3 , and $f(x)=g(x)=1 /\left(1+e^{-x}\right)-1 / 2$. Obviously, $L^{f}=L^{g}=$ 0.25 . The initial condition is given as $x(t)=-0.43, y(t)=$ $0.42, t \in[-3,0]$. With the above system parameters, the phase diagram of system (75) is given in Figure 2. Obviously, the origin of system (75) is not stable. We will design the sliding intermittent controller to stabilize it.

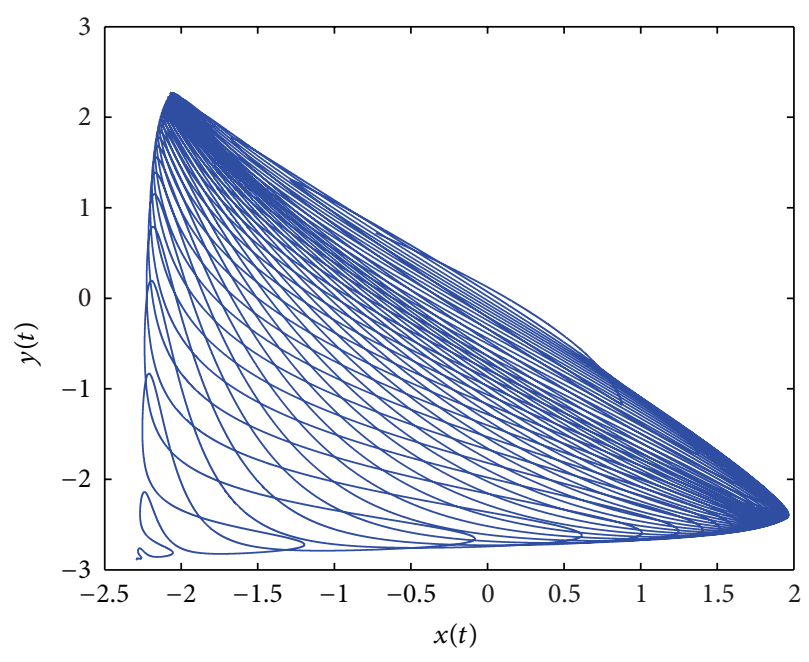

FIGURE 2: The phase diagram of the original system (75).

Applying the sliding intermittent controller to the unstable system (75), one can derive the following system:

$$
\begin{aligned}
& \dot{x}(t)=-a x(t)+p f(y(t-\tau))+k_{1} x(t), t \in[l T,(l+\theta) T) \\
& \dot{x}(t)=-a x(t)+p f(y(t-\tau)), \\
& \quad t \in[(l+\theta) T,(l+1) T), t \neq t_{k}, \\
& \Delta x\left(t_{k}\right)=h_{1} x\left(t_{k}\right), \quad t=t_{k}, \\
& \dot{y}(t)=-b y(t)+q g(x(t-\sigma))+k_{2} y(t), \\
& \quad t \in[l T,(l+\theta) T), \\
& \dot{y}(t)=-b y(t)+q g(x(t-\sigma)), \\
& t \in[(l+\theta) T,(l+1) T), t \neq t_{k}, \\
& \Delta y\left(t_{k}\right)=h_{2} y\left(t_{k}\right), \quad t=t_{k} .
\end{aligned}
$$

In the following, we will give the convergence results by simulating the system (76). Firstly, by setting the continuous feedback gains $k_{1}=-4.8512, k_{2}=-4.6378$ and the impulsive strengths $h_{1}=h_{2}=-0.15$. With the above parameters setting, calculations show that $\eta^{*}=0.2679$ and $\mu=0.85$. By setting the control period $T=10$, we have control width $0.3<\theta<0.7$ and $\gamma=0.3$. Here we take the parameter $\theta=0.5$. If we utilize uniform distributed impulsive sequences $\left(t_{k}-t_{k-1}=0.01\right)$ in the latter control width of the control period, it is easy to check that when $\eta=$ 0.0221 , we have $\rho=-1.1410, \widetilde{a}_{1}=5.7788$, and $\tilde{b}_{1}=4.8302$. By choosing $\beta=-4.8519$, one has $d=1.1765, \ln \mu /\left(t_{k}-\right.$ $\left.t_{k-1}\right)-\beta=-11.4000, \rho+d \tilde{b}_{1}+\beta=-0.3104, \lambda_{1}=$ $0.0565, \lambda_{2}=0.0168$, and $\eta+\lambda_{1}(\theta-\gamma)-\lambda_{2}(\theta+\gamma)-\ln d / T=$ 0.0037 , which means all the conditions in Theorem 6 hold. The simulation result under the sliding intermittent control is given in Figure 3. 


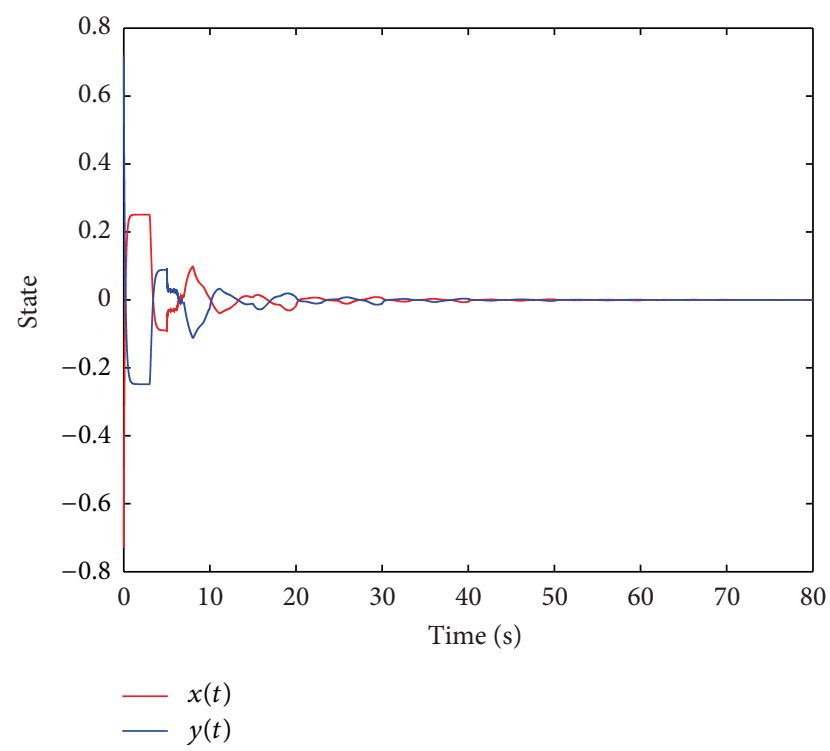

FIGURE 3: Simulation of the system (75) under the sliding intermittent control.

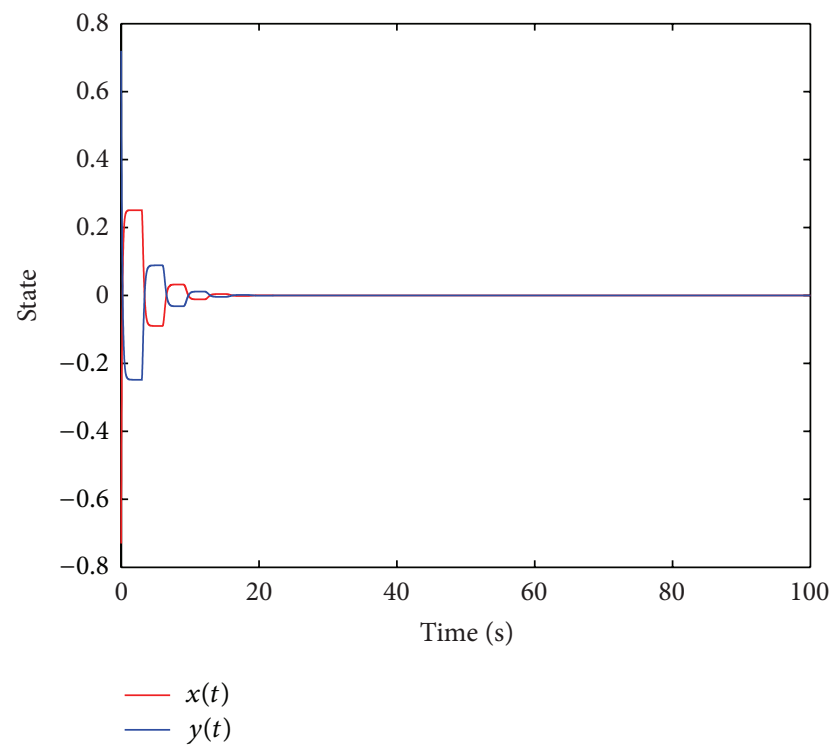

Figure 4: Simulation of the system (75) under the continuous feedback control.

Next, the corresponding convergence results under the propositions obtained in this article will be illustrated.

If we utilize the continuous feedback control, by taking the same continuous feedback gains as the above sliding intermittent control, the conditions in Proposition 9 hold, and the simulation result under the continuous feedback control is given in Figure 4.

If we use pure periodically intermittent control, by setting the continuous feedback gains $k_{1}=-6.8512, k_{2}=-6.6378$, we get $\eta^{*}=0.3682, \widetilde{\rho}=\kappa=0.0001$. It is easy to know when $\eta=0.0002$, we have $\widetilde{a}_{1}=7.8007, \widetilde{b}_{1}=4.5230$, and $\lambda_{1}=0.1742$. In Proposition 11, by taking $T=100$,

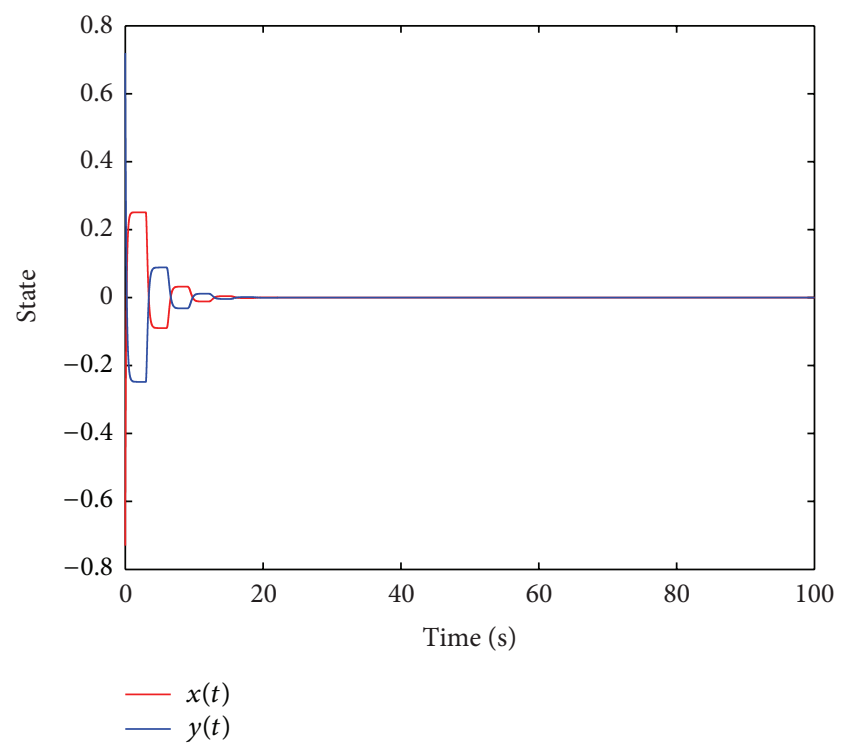

Figure 5: Simulation of the system (75) under the periodically intermittent control.

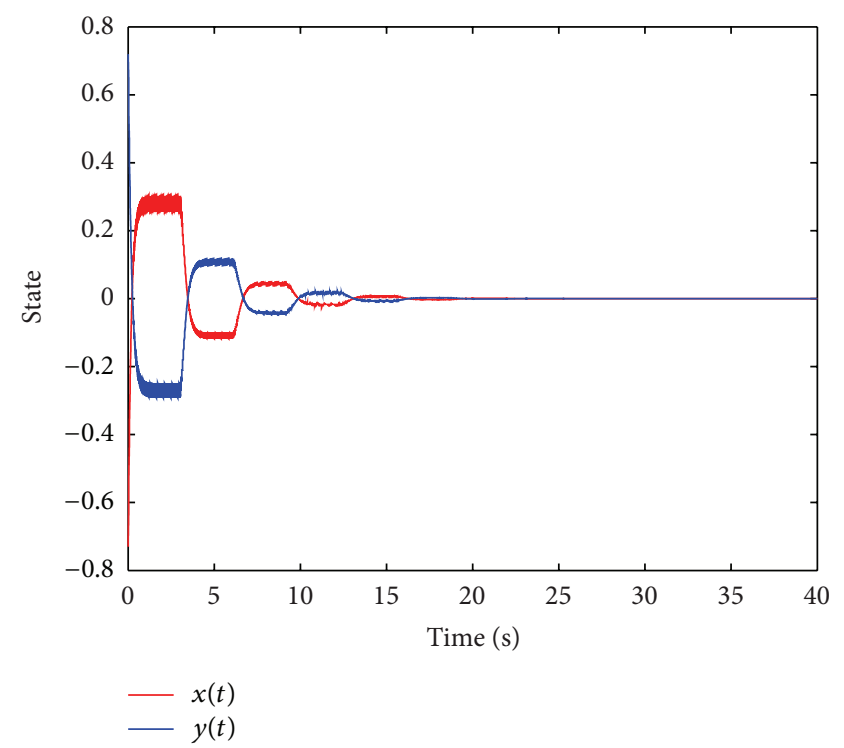

FIGURE 6: Simulation of the system (75) under the full impulsive control.

the relationships among the upper bound delay, the control width, and the control period infer that $0.03<\theta<0.97$, while the last inequality in Proposition 11 means $\theta>0.9640$. Here we set the $\theta=0.9650$, and the simulation result under the periodically intermittent control is given in Figure 5.

If we use full impulsive control with uniform distributed impulsive sequences $\left(t_{k}-t_{k-1}=0.04\right)$ and the impulsive strengths $\mu_{k}=0.85, k=1,2, \ldots$, it is easy to check when $\beta=$ $-3.1262, \eta=0.0002$, the parameters $d=1.1332, \rho=$ -1.1629 , and $\widetilde{b}_{1}=4.5230$, all the conditions in Proposition 12 satisfied. If we use the semi-impulsive control scheme and set the impulsive sequences satisfying the average impulsive 


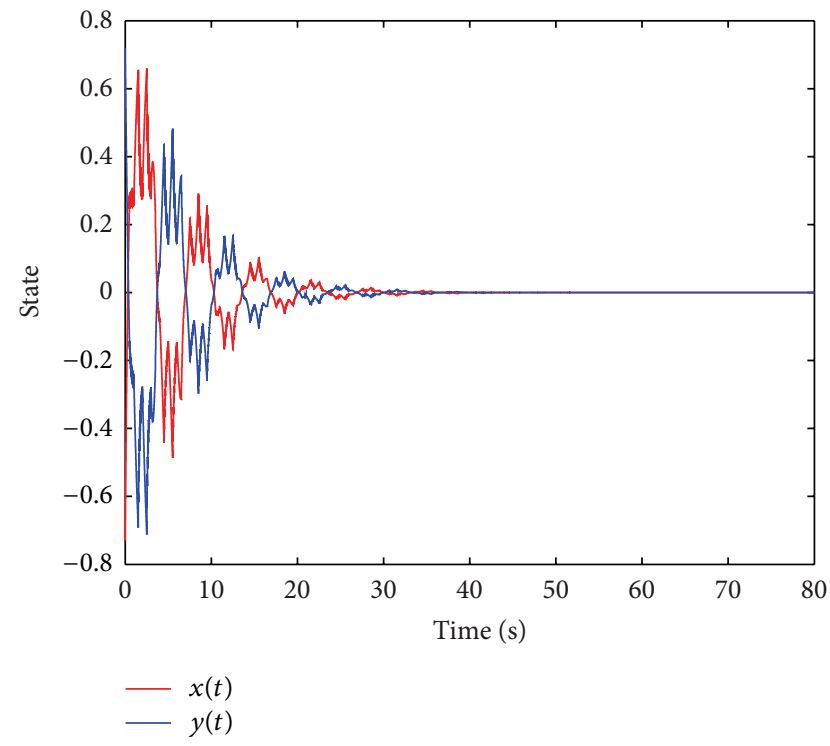

FIGURE 7: Simulation of the system (75) under the semi impulsive control.

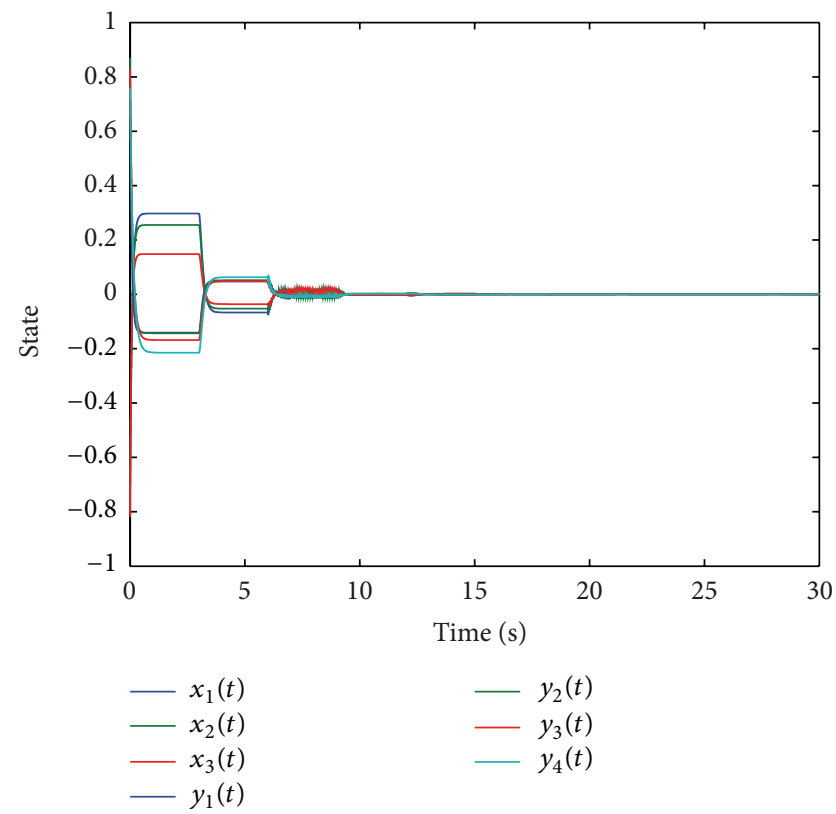

FIGURE 8: Simulation of the system (77) under the sliding intermittent controller.

interval with $T_{a}=0.038$ and $N_{0}=1$; it is easy to check $\rho+$ $\left(\ln \mu / T_{a}\right)+\mu^{-N_{0}} \widetilde{b}_{1}=-0.1185<0$, which infers that the condition in Proposition 16 holds. The simulation results for system (75) with the full impulsive control and the semiimpulsive control are given in Figures 6 and 7.

Remark 18. From the above verifying process, it can be found that the sliding intermittent control is much better than the pure periodically intermittent control. More specifically, in the periodically intermittent control, the control period $T=$ 100 and the control width $\theta=0.965$, while in the sliding intermittent control, the control period $T=10$ and the control width $\theta=0.5$. As for the impulsive control, the full impulsive control is better than the semi-impulsive control in that the earlier converges faster. When dealing with the nonuniformly distributed impulsive sequence, the result derived in Proposition 16 is less conservative.

Example 2. Consider the following unstable delayed BAM neural network, and we will show that the sliding intermittent control benefits the stabilization of the unstable system:

$$
\begin{aligned}
& \dot{x}_{i}(t)=-a_{i} x_{i}(t)+\sum_{j=1}^{3} p_{i j} f_{j}\left(y_{j}\left(t-\tau_{i j}\right)\right), \quad i \in \mathscr{I}_{3}, \\
& \dot{y}_{j}(t)=-b_{j} y_{j}(t)+\sum_{i=1}^{4} q_{j i} g_{i}\left(x_{i}\left(t-\sigma_{j i}\right)\right), \quad j \in \mathscr{I}_{4},
\end{aligned}
$$

where $A=\operatorname{diag}\{3.1220,2.3156,2.2683\}, B=\operatorname{diag}\{2.9631$, $2.3456,2.6341,3.0726\}$,

$$
\begin{gathered}
P=\left[\begin{array}{llll}
7.8501 & 2.3070 & 3.2280 & 4.7191 \\
3.2463 & 6.0589 & 5.3751 & 2.2609 \\
2.0159 & 1.7803 & 2.6601 & 5.7647
\end{array}\right], \\
Q=\left[\begin{array}{lll}
4.2331 & 4.3741 & 2.2459 \\
4.8830 & 2.3259 & 1.2857 \\
2.4022 & 1.3377 & 3.7930 \\
2.1351 & 2.6759 & 3.4719
\end{array}\right] .
\end{gathered}
$$

The activations functions $f(x)=g(x)=1 /(1+$ $\left.e^{-x}\right)-1 / 2$ with $L^{f}=L^{g}=0.25$ and the time delays $\tau=$ $\sigma=3$. The system has an unstable equilibrium 0 under the above parameters with the initial functions $x(t)=$ $[-0.73,-0.79,-0.82]^{T}, y(t)=[0.72,0.87,0.83,0.76]^{T}, t \in$ $[-3,0]$.

In the following, we will check the convergence results for system (77) under the sliding intermittent controller. Setting the continuous feedback gains $k^{(1)}=$ $\operatorname{diag}\{-8.0350,-10.4875,-13.1075\}, k^{(2)}=\operatorname{diag}\{-11.1950$, $-8.4125,-5.7425,-4.1475\}$, the impulsive strengths are identical with $h_{i k}^{(1)}=h_{j k}^{(2)}=-0.25, i \in \mathscr{I}_{n}, j \in \mathscr{I}_{m}, k \in \mathbb{N}^{+}$, and the control period $T=15$; some calculations show that $\eta^{*}=0.2604, \mu=0.75$. We can get the control width $0.2<\theta<0.8$ and $\gamma=0.2$; here we take the parameter $\theta=0.4$. If we utilize the uniform distributed impulsive sequences $\left(t_{k}-t_{k-1}=0.02\right)$ in the latter control width of the control period, it is easy to know when $\eta=$ 0.1250, we have $\rho=-2.1433, \widetilde{a}_{1}=7.0951$, and $\widetilde{b}_{1}=4.9664$. By choosing $\beta=-4.6841$, one has $\ln \mu /\left(t_{k}-t_{k-1}\right)-\beta=$ $-9.7000, d=1.333, \rho+d \widetilde{d}_{1}+\beta=-0.2055, \lambda_{1}=0.1135, \lambda_{2}=$ 0.1924 , and $\eta+\lambda_{1}(\theta-\gamma)-\lambda_{2}(\theta+\gamma)-\ln d / T=0.0131$, which mean all the conditions in Theorem 6 hold. The simulation result under the sliding intermittent control is given in Figure 8.

\section{Conclusions}

In this paper, a sliding intermittent controller has been proposed by unifying the periodically intermittent control 
with the impulsive control together with continuous feedback control. More specifically, the continuous feedback control is employed as the preceding control width, and the impulsive control is resorted in the latter control width. Furthermore, the adjustable parameter $\theta \in[0,1]$ is very flexible in that the continuous feedback control $(\theta=1)$, the impulsive control $(\theta=0)$, the periodically intermittent control, and the semiimpulsive control $(0<\theta<1)$ are all possible cases. Based on the analysis technique and the Lyapunov function approach, the conditions have been constructed for the exponential stability of the delayed BAM neural networks under the proposed control schemes. Finally, numerical simulations are used to illustrate the effectiveness of the control technique.

\section{Acknowledgments}

This work was supported in part by the National Natural Science Foundation of China under Grant 61174136, the Natural Science Foundation of Jiangsu Province of China under Grant BK2011598, the Program for New Century Excellent Talents in University under Grant NCET-12-0117, and the Fundamental Research Funds for the Central Universities under Grant 2242012155.

\section{References}

[1] B. Kosko, "Bidirectional associative memories," IEEE Transactions on Systems, Man, and Cybernetics, vol. 18, no. 1, pp. 49-60, 1988.

[2] V. S. H. Rao and B. R. M. Phaneendra, "Global dynamics of bidirectional associative memory neural networks involving transmission delays and dead zones," Neural Networks, vol. 12, no. 3, pp. 455-465, 1999.

[3] J. Cao, J. Liang, and J. Lam, "Exponential stability of high-order bidirectional associative memory neural networks with time delays," Physica D, vol. 199, no. 3-4, pp. 425-436, 2004.

[4] Y. Liu, Z. Wang, J. Liang, and X. Liu, "Synchronization of coupled neutral-type neural networks with jumping-modedependent discrete and unbounded distributed delays," IEEE Transactions on Cybernetics, vol. 43, no. 1, pp. 102-114, 2012.

[5] J. Liang, Z. Wang, B. Shen, and X. Liu, "Distributed state estimation in sensor networks with randomly occurring nonlinearities subject to time delays," ACM Transactions on Sensor Networks, vol. 9, no. 1, article 4, 2012.

[6] D. Ding, Z. Wang, H. Dong, and H. Shu, "Distributed $H_{\infty}$ state estimation with stochastic parameters and nonlinearities through sensor networks: the finite-horizon case," Automatica, vol. 48, no. 8, pp. 1575-1585, 2012.

[7] M. Xiao, W. X. Zheng, and J. Cao, "Hopf bifurcation of an ( $n$ +1 )-neuron bidirectional associative memory neural network model with delays," IEEE Transactions on Neural Networks and Learning Systems, vol. 24, no. 1, pp. 118-132, 2013.

[8] K. Gopalsamy and X.-Z. He, "Delay-independent stability in bidirectional associative memory networks," IEEE Transactions on Neural Networks, vol. 5, no. 6, pp. 998-1002, 1994.

[9] S. Arik, "Global asymptotic stability analysis of bidirectional associative memory neural networks with time delays," IEEE Transactions on Neural Networks, vol. 16, no. 3, pp. 580-586, 2005.
[10] J. Liang and J. Cao, "Exponential stability of continuous-time and discrete-time bidirectional associative memory networks with delays," Chaos, Solitons \& Fractals, vol. 22, no. 4, pp. 773$785,2004$.

[11] J. Cao and Q. Song, "Stability in Cohen-Grossberg-type bidirectional associative memory neural networks with time-varying delays," Nonlinearity, vol. 19, no. 7, pp. 1601-1617, 2006.

[12] J. Lunze and D. Lehmann, "A state-feedback approach to eventbased control," Automatica, vol. 46, no. 1, pp. 211-215, 2010.

[13] C. A. S. Batista, S. R. Lopes, R. L. Viana, and A. M. Batista, "Delayed feedback control of bursting synchronization in a scale-free neuronal network," Neural Networks, vol. 23, no. 1, pp. 114-124, 2010.

[14] J. Lu and J. Cao, "Adaptive synchronization in tree-like dynamical networks," Nonlinear Analysis. Real World Applications, vol. 8, no. 4, pp. 1252-1260, 2007.

[15] J. Liang, Z. Wang, and X. Liu, "On passivity and passification of stochastic fuzzy systems with delays: the discrete-time case," IEEE Transactions on Systems, Man, and Cybernetics B, vol. 40, no. 3, pp. 964-969, 2010.

[16] B. Shen, Z. Wang, and X. Liu, "Sampled-data synchronization control of dynamical networks with stochastic sampling," IEEE Transactions on Automatic Control, vol. 57, no. 10, pp. 26442650, 2012.

[17] L. Wu, P. Shi, and H. Gao, "State estimation and slidingmode control of Markovian jump singular systems," IEEE Transactions on Automatic Control, vol. 55, no. 5, pp. 1213-1219, 2010.

[18] J. Hu, Z. Wang, H. Gao, and L. K. Stergioulas, "Extended Kalman filtering with stochastic nonlinearities and multiple missing measurements," Automatica, vol. 48, no. 9, pp. 20072015, 2012.

[19] G. Wei, Z. Wang, and B. Shen, "Probability-dependent gainscheduled control for discrete stochastic delayed systems with randomly occurring nonlinearities," International Journal of Robust and Nonlinear Control, vol. 23, no. 7, pp. 815-826, 2012.

[20] L. Pan and J. Cao, "Exponential stability of impulsive stochastic functional differential equations," Journal of Mathematical Analysis and Applications, vol. 382, no. 2, pp. 672-685, 2011.

[21] J. Lu, D. W. C. Ho, and J. Cao, "A unified synchronization criterion for impulsive dynamical networks," Automatica, vol. 46, no. 7, pp. 1215-1221, 2010.

[22] C. Li, G. Feng, and X. Liao, "Stabilization of nonlinear systems via periodically intermittent control," IEEE Transactions on Circuits and Systems II, vol. 54, no. 11, pp. 1019-1023, 2007.

[23] W. Xia and J. Cao, "Pinning synchronization of delayed dynamical networks via periodically intermittent control," Chaos, vol. 19, no. 1, Article ID 013120, 2009.

[24] D. S. Goshi, K. M. K. H. Leong, and T. Itoh, "A secure highspeed retrodirective communication link," IEEE Transactions on Microwave Theory and Techniques, vol. 53, no. 11, pp. 35483556, 2005.

[25] D. W. C. Ho, J. Liang, and J. Lam, "Global exponential stability of impulsive high-order BAM neural networks with time-varying delays," Neural Networks, vol. 19, no. 10, pp. 1581-1590, 2006.

[26] Q. Gan, "Exponential synchronization of stochastic CohenGrossberg neural networks with mixed time-varying delays and reaction-diffusion via periodically intermittent control," Neural Networks, vol. 31, pp. 12-21, 2012.

[27] S. Mohamad, "Global exponential stability in continuoustime and discrete-time delayed bidirectional neural networks," Physica D, vol. 159, no. 3-4, pp. 233-251, 2001. 
[28] R. Samidurai, R. Sakthivel, and S. M. Anthoni, "Global asymptotic stability of BAM neural networks with mixed delays and impulses," Applied Mathematics and Computation, vol. 212, no. 1, pp. 113-119, 2009.

[29] Y. Li and C. Wang, "Existence and global exponential stability of equilibrium for discrete-time fuzzy BAM neural networks with variable delays and impulses," Fuzzy Sets and Systems, vol. 217, pp. 62-79, 2013.

[30] A. Halanay, Differential Equations: Stability, Oscillations, Time Lags, Academic Press, New York, NY, USA, 1966.

[31] L. Pan and J. Cao, "Anti-periodic solution for delayed cellular neural networks with impulsive effects," Nonlinear Analysis. Real World Applications, vol. 12, no. 6, pp. 3014-3027, 2011.

[32] Y.-T. Li and C.-B. Yang, "Global exponential stability analysis on impulsive BAM neural networks with distributed delays," Journal of Mathematical Analysis and Applications, vol. 324, no. 2, pp. 1125-1139, 2006.

[33] V. Lakshmikantham, D. D. Bainnov, and P. S. Simeonov, Theory of impulsive differential equations, vol. 6 of Series in Modern Applied Mathematics, World Scientific Publishing Company, Teaneck, NJ, USA, 1989.

[34] L.-J. Pan, G.-Q. Wang, and S. S. Cheng, "Oscillation of even order nonlinear differential equations with impulses," Funkcialaj Ekvacioj. Serio Internacia, vol. 50, no. 1, pp. 117-131, 2007.

[35] X. Liu and T. Chen, "Cluster synchronization in directed networks via intermittent pinning control," IEEE Transactions on Neural Networks, vol. 22, no. 7, pp. 1009-1020, 2011.

[36] H. Dong, Z. Wang, and H. Gao, "Fault detection for Markovian jump systems with sensor saturations and randomly varying nonlinearities," IEEE Transactions on Circuits and Systems I, vol. 59, no. 10, pp. 2354-2362, 2012.

[37] J. Hu, Z. Wang, B. Shen, and H. Gao, "Gain-constrained recursive filtering with stochastic nonlinearities and probabilistic sensor delays," IEEE Transactions on Signal Processing, vol. 61, no. 5, pp. 1230-1238, 2013.

[38] B. Shen, S. X. Ding, and Z. Wang, "Finite-horizon $H_{\infty}$ fault estimation for linear discrete time-varying systems with delayed measurements," Automatica, vol. 49, no. 1, pp. 293-296, 2013.

[39] H. Dong, Z. Wang, J. Lam, and H. Gao, "Fuzzy-model-based robust fault detection with stochastic mixed time delays and successive packet dropouts," IEEE Transactions on Systems, Man, and Cybernetics B, vol. 42, no. 2, pp. 365-376, 2012. 


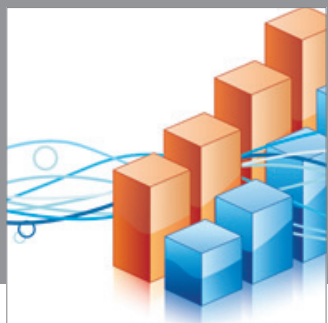

Advances in

Operations Research

mansans

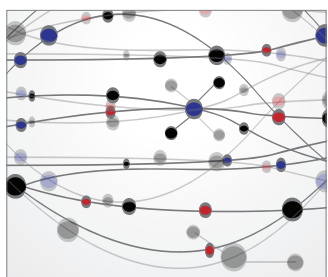

The Scientific World Journal
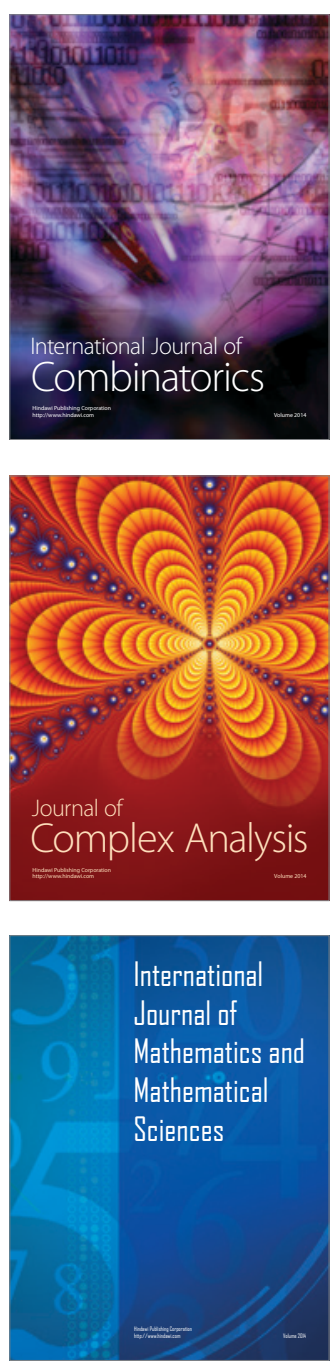
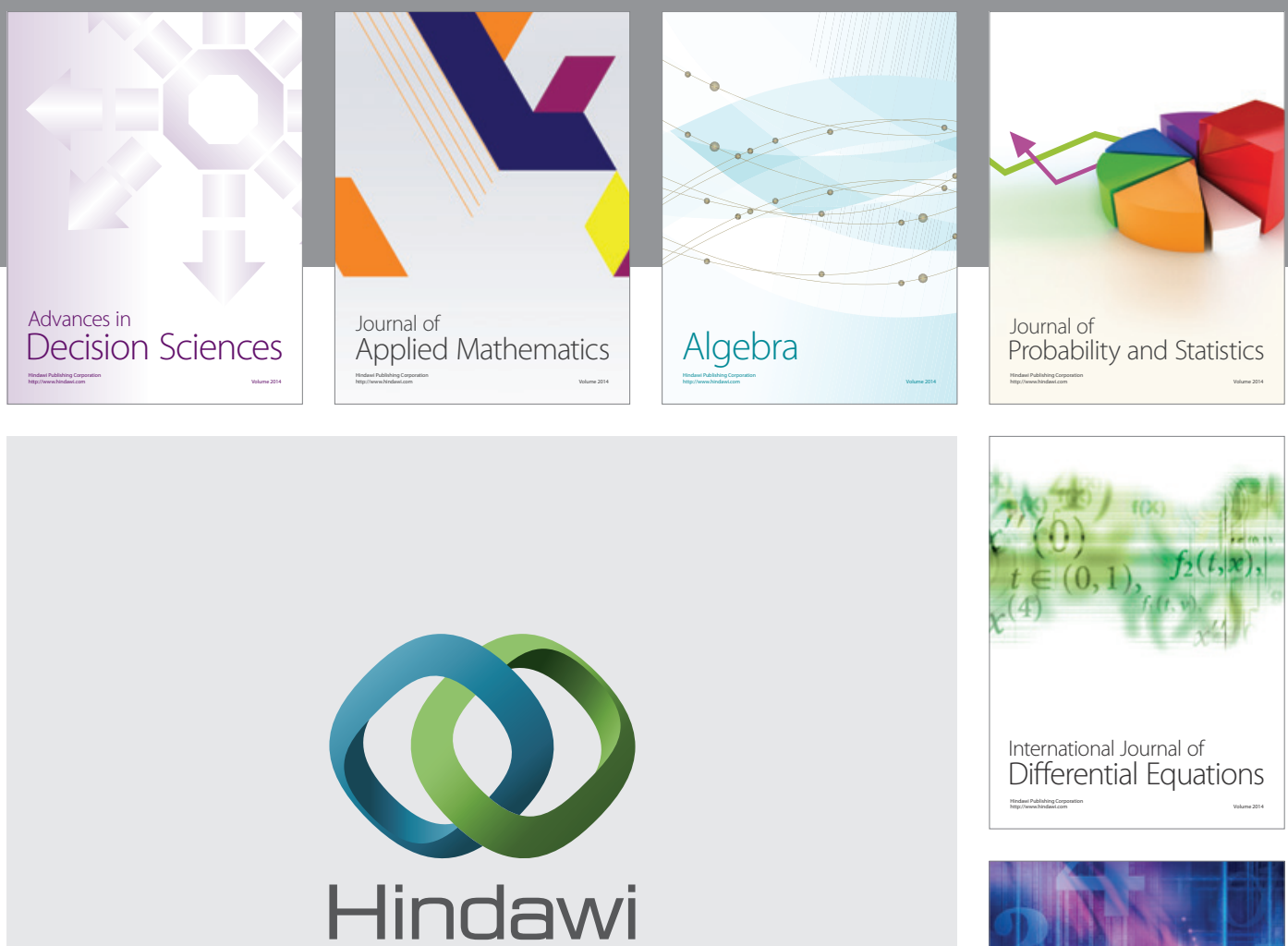

Submit your manuscripts at http://www.hindawi.com
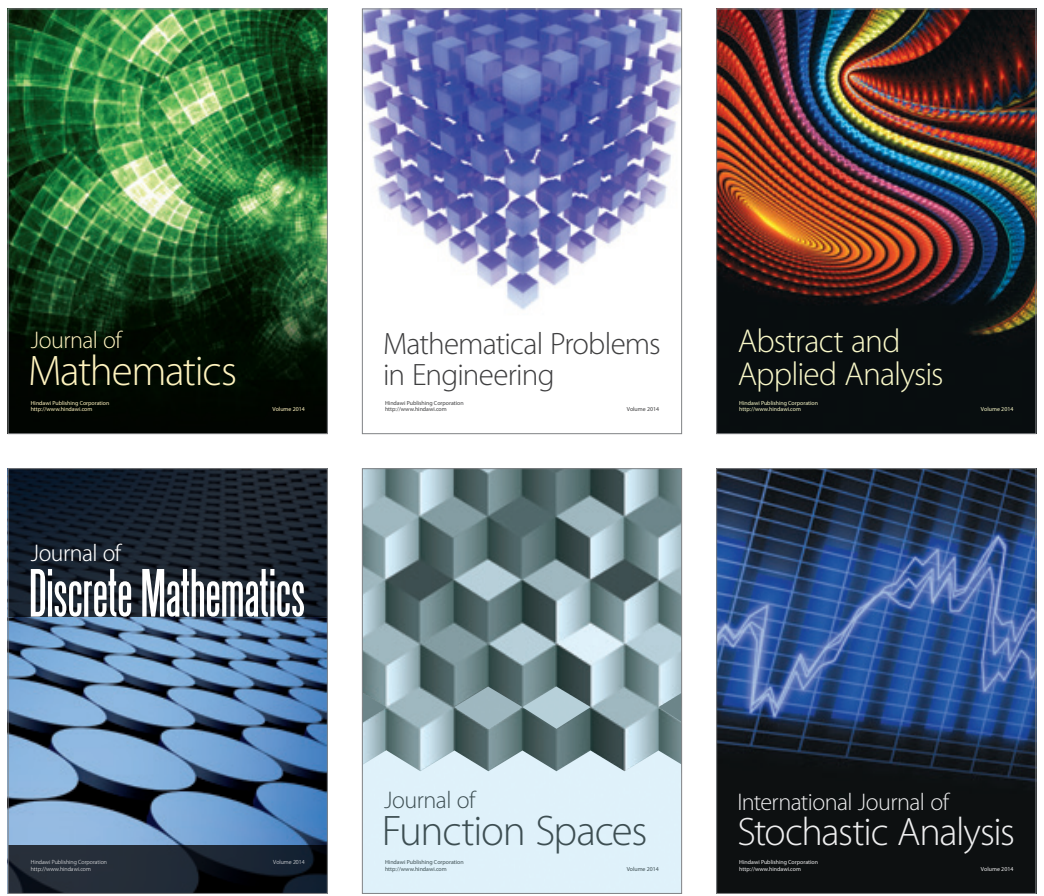

Journal of

Function Spaces

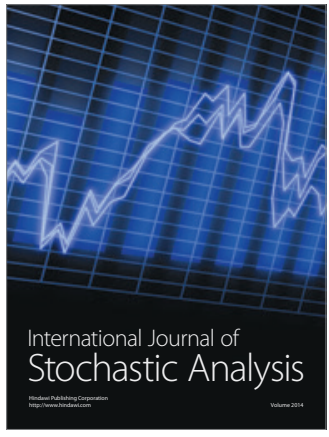

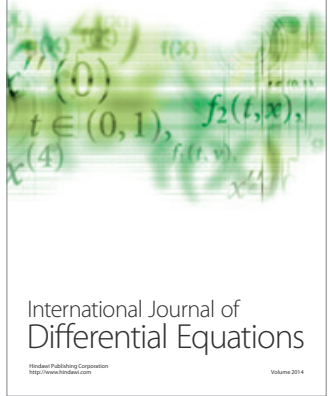
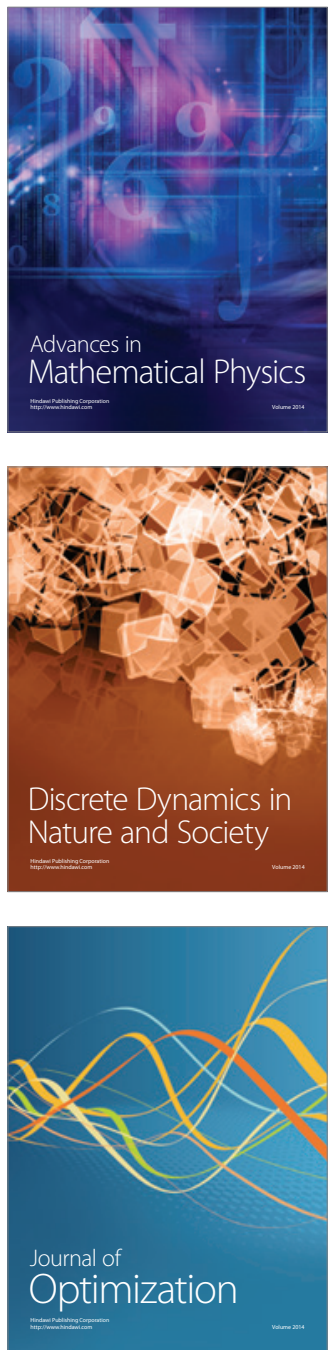